\title{
LOCAL GROUP DWARF ELLIPTICAL GALAXIES. II. STELLAR KINEMATICS TO LARGE RADII IN NGC 147 AND NGC 185
}

\author{
M. Geha ${ }^{1}$, R. P. van der Marel ${ }^{2}$, P. Guhathakurta ${ }^{3}$, K. M. Gilbert ${ }^{4}$, J. Kalirai ${ }^{2}$, and E. N. Kirby ${ }^{5,6}$ \\ ${ }^{1}$ Astronomy Department, Yale University, New Haven, CT 06520, USA; marla.geha@yale.edu \\ ${ }^{2}$ Space Telescope Science Institute, 3700 San Martin Drive, Baltimore, MD 21218, USA \\ ${ }^{3}$ UCO/Lick Observatory, University of California, Santa Cruz, 1156 High Street, Santa Cruz, CA 95064, USA \\ ${ }^{4}$ Department of Astronomy, University of Washington, Seattle, WA 98195-1580, USA \\ ${ }^{5}$ California Institute of Technology, Department of Astronomy, 1200 E California Blvd, Pasadena, CA 91125, USA \\ Received 2009 July 30; accepted 2010 January 6; published 2010 February 10
}

\begin{abstract}
We present kinematic and metallicity profiles for the M 31 dwarf elliptical (dE) satellite galaxies NGC 147 and NGC 185. The profiles represent the most extensive spectroscopic radial coverage for any dE galaxy, extending to a projected distance of 8 half-light radii $\left(8 r_{\text {eff }} \sim 14^{\prime}\right)$. We achieve this coverage via Keck/DEIMOS multislit spectroscopic observations of 520 and 442 member red giant branch stars in NGC 147 and NGC 185, respectively. In contrast to previous studies, we find that both dEs have significant internal rotation. We measure a maximum rotational velocity of $17 \pm 2 \mathrm{~km} \mathrm{~s}^{-1}$ for NGC 147 and $15 \pm 5 \mathrm{~km} \mathrm{~s}^{-1}$ for NGC 185 . While both rotation profiles suggest a flattening in the outer regions, there is no indication that we have reached the radius of maximum rotation velocity. The velocity dispersions decrease gently with radius with average dispersions of $16 \pm 1 \mathrm{~km} \mathrm{~s}$ and $24 \pm 1 \mathrm{~km} \mathrm{~s}^{-1}$ for NGC 147 and NGC 185, respectively. The average metallicities for NGC 147 and NGC 185 are $[\mathrm{Fe} / \mathrm{H}]=-1.1 \pm 0.1$ and $[\mathrm{Fe} / \mathrm{H}]=-1.3 \pm 0.1$, respectively; both $\mathrm{dEs}$ have internal metallicity dispersions of $0.5 \mathrm{dex}$, but show no evidence for a radial metallicity gradient. We construct two-integral axisymmetric dynamical models and find that the observed kinematical profiles cannot be explained without modest amounts of non-baryonic dark matter. We measure central mass-to-light ratios of $M / L_{V}=4.2 \pm 0.6$ and $M / L_{V}=4.6 \pm 0.6$ for NGC 147 and NGC 185, respectively. Both dE galaxies are consistent with being primarily flattened by their rotational motions, although some anisotropic velocity dispersion is needed to fully explain their observed shapes. The velocity profiles of all three Local Group dEs (NGC 147, NGC 185, and NGC 205) suggest that rotation is more prevalent in the $\mathrm{dE}$ galaxy class than previously assumed, but often manifests only at several times the effective radius. Since all dEs outside the Local Group have been probed to only inside the effective radius, this opens the door for formation mechanisms in which dEs are transformed or stripped versions of gas-rich rotating progenitor galaxies.
\end{abstract}

Key words: galaxies: dwarf - galaxies: individual (NGC 147, NGC 185) - galaxies: kinematics and dynamics

Online-only material: color figures, machine-readable tables

\section{INTRODUCTION}

Dwarf elliptical (dE) galaxies are characterized by low surface brightness $\left(\mu_{\text {eff }, V}>22 \mathrm{mag} \operatorname{arcsec}^{-2}\right)$, little to no gas, and old to intermediate age stellar populations. These galaxies are spatially clustered and account for more than $75 \%$ of objects brighter than $M_{V}<-14$ in nearby galaxy clusters (Ferguson \& Sandage 1991; Ferguson \& Binggeli 1994). Since there are few, if any, isolated $\mathrm{dE}$ galaxies, most proposed $\mathrm{dE}$ galaxy formation scenarios are environmentally driven, such as via gas stripping or gravitational harassment of gas-rich spiral or dwarf irregular galaxies (e.g. Dekel \& Silk 1986; Moore et al. 1998; Aguerri \& González-García 2009). Recent evidence for embedded stellar disks and bars in some dEs support these scenarios (Jerjen et al. 2000; Geha et al. 2005; Lisker et al. 2006; Chilingarian et al. 2007). Since gas-rich systems have significant rotation velocities, a key test of this hypothesis class is comparing the kinematics of $\mathrm{dE}$ and gas-rich galaxies.

Internal kinematic measurements for dEs in the Fornax and Virgo Cluster have revealed an unexpected dichotomy: roughly half of the observed dEs have significant rotation about the major-axis, while the remaining galaxies show no detectable major-axis rotation (Pedraz et al. 2002; Geha et al. 2003; van Zee et al. 2004; de Rijcke et al. 2005). dE galaxies with no rotation present a challenge to any scenario in which $\mathrm{dE}$ progenitors

\footnotetext{
6 Hubble Fellow
}

are gas-rich and thus rotating (Moore et al. 1998). Ram pressure stripping alone does not affect the kinematics of the collisionless stellar component. Gravitational processes such as galaxy harassment can reduce the amount of rotational angular momentum, converting this into random velocity dispersion; however, simulations are unable to completely remove rotational support (Mayer et al. 2001; Mastropietro et al. 2005).

Because dEs have low surface brightnesses, integrated light (long-slit) kinematic observations of dEs in both galaxy clusters and the Local Group are currently limited to within the halflight effective radius $\left(r_{\text {eff }}\right)$. Thus, it is possible that significant rotational angular momentum lies at larger distances than has so far been explored. Addressing this question requires kinematics tracers at larger radius. While the dynamics of $\mathrm{dE}$ globular cluster systems suggest that there may be significant rotation at large radius (Beasley et al. 2006, 2009), the small number of clusters limits the usefulness of this tracer. The dEs in the Local Group share the same general properties as the more numerous dEs in galaxy clusters; however, their proximity allows us to resolve individual stars (Geha et al. 2006), and thus trace kinematics profiles down to arbitrarily low surface brightness and large radius.

The Local Group contains three dE galaxies, NGC 205, NGC 147, and NGC 185, which in addition to the compact elliptical M32 are the brightest satellites around the spiral galaxy M 31. In our terminology of ellipsoidal systems, we follow the 
Table 1

Local Group dEs at a Glance

\begin{tabular}{|c|c|c|c|c|c|c|c|c|c|}
\hline Name & $\begin{array}{c}\alpha(\mathrm{J} 2000) \\
(\mathrm{h}: \mathrm{m}: \mathrm{s})\end{array}$ & $\begin{array}{c}\delta(\mathrm{J} 2000) \\
\left({ }^{\circ}:^{\prime}:^{\prime \prime}\right)\end{array}$ & Type & $\begin{array}{l}\text { Dist. } \\
(\mathrm{kpc})\end{array}$ & $m_{V}$ & $A_{V}$ & $M_{V, 0}$ & $\epsilon$ & 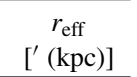 \\
\hline NGC 147 & $00: 33: 12$ & $+48: 30: 31$ & $\mathrm{dE} 5$ & $675 \pm 27$ & $9.52 \pm 0.07$ & 0.57 & -15.5 & 0.44 & $2.03(0.40)$ \\
\hline NGC 185 & $00: 38: 58$ & $+48: 20: 14$ & dE3 & $616 \pm 26$ & $9.18 \pm 0.05$ & 0.61 & -15.7 & 0.23 & $1.50(0.27)$ \\
\hline NGC 205 & $00: 40: 22$ & $+41: 41: 07$ & dE5 & $824 \pm 27$ & $8.07 \pm 0.07$ & 0.21 & -16.7 & 0.50 & $2.17(0.52)$ \\
\hline
\end{tabular}

Notes. The right ascension, declination, and morphological type are take from NASA/IPAC Extragalactic Database (NED). We adopt the distances determined by McConnachie et al. (2005). The total apparent $V$-band magnitude for each dE is taken from the RC 3 catalog. The absolute magnitude is calculated assuming an extinction values from Schlegel et al. (1998). The ellipticity is the average value between $1^{\prime}-5^{\prime}$ from Kent (1987). The effective, half-light radii are from de Rijcke et al. (2006), based on near-infrared 2MASS photometry.

conventions of, e.g., Bender et al. (1992), although alternative terminologies are in use. While the differing terminologies can be confusing at times, the key point is that NGC 205, NGC 147, and NGC 185 on the one hand, and M 32 on the other hand, should not be grouped in a single class. The former three galaxies have fundamental plane properties that indicate similarity to galaxies in the dwarf spheroidal class (dSph). By contrast, galaxies such as M 32 have properties that indicate similarity to (giant) elliptical galaxies. This likely indicates a difference in formation history, as discussed more fully in, e.g., Kormendy et al. (2009).

The prototype of the dE galaxy class is NGC 205 which is in very close projection to $\mathrm{M} 31\left(40^{\prime}=8 \mathrm{kpc}\right)$. Both photometric and kinematic evidence suggest that NGC 205 is tidally interacting with M 31 (Choi et al. 2002; Geha et al. 2006) and may in fact be on its first orbital approach (Howley et al. 2008). In contrast, NGC 147 and NGC 185 lie at a projected distance of $7^{\circ}(\sim 150 \mathrm{kpc})$ from M 31. While the metal-poor stellar halo of M 31 extends to this distance (Guhathakurta et al. 2005; Kalirai et al. 2006), its gravitational influence of M 31 is not expected to influence the kinematics of these $\mathrm{dE}$ satellites (see Section 4.5). NGC 147 and NGC 185 are separated from each other by merely $58^{\prime}$, leading to speculation that they may be a bound galaxy pair (Baade 1944; van den Bergh 1998). An accurate distance estimate suggests that these two galaxies are physically separated by over $60 \mathrm{kpc}$ (McConnachie et al. 2005) and may not be bound (however, see Section 4.5). The large observed distances between these two dEs and their parent M 31, combined with their relative proximity to the Sun, make NGC 147 and NGC 185 the best available targets for studying the properties of $\mathrm{dE}$ galaxies to large radius.

NGC 147 and NGC 185 share several similar fundamental properties, such as absolute luminosity $\left(M_{V} \sim-15.5\right)$, halflight radius $\left(r_{\text {eff }} \sim 2^{\prime}=0.3 \mathrm{kpc}\right)$, and central velocity dispersion (for exact values see Table 1). However, they differ markedly in many aspects. NGC 185 contains some gas, dust, and evidence for recent star formation confined to its center (MartínezDelgado et al. 1999), while NGC 147 is devoid of gas or dust and shows no sign of recent star formation activity (Young \& Lo 1997; Sage et al. 1998). An average metallicity of $[\mathrm{Fe} / \mathrm{H}]=$ -1.4 is inferred for NGC 185 (Martínez-Delgado et al. 1999), while a more metal-rich population of $[\mathrm{Fe} / \mathrm{H}]=-1.0$ is inferred for NGC 147 (Han et al. 1997; Davidge 2005; Gonçalves et al. 2007). Hubble Space Telescope/Wide Field Planetary Camera 2 (HST/WFPC2) imaging of both galaxies also implies the presence of intermediate-age stars, with NGC 147 having a more significant contribution than NGC 185 (Butler \& MartínezDelgado 2005). Thus, while both dEs are dominated by old- to intermediate-age stars, the metallicity and age mixture of these components are different in each galaxy.
The first spectroscopic long-slit observations of Local Group dEs by Bender et al. (1991) suggested that while NGC 185 appeared supported entirely by random motions, NGC 147 had a significant rotational component of $6.5 \pm 1.1 \mathrm{~km} \mathrm{~s}^{-1}$. Somewhat deeper observations by Simien \& Prugniel (2002) extended the kinematic profiles out $\sim 2^{\prime}\left(1 r_{\text {eff }}\right)$, but did not measure a significant rotation for either galaxy. In contrast, both studies measured a rotational component in NGC 205 (within the tidal radius), later confirmed by Geha et al. (2006) to be $11 \pm 5 \mathrm{~km} \mathrm{~s}^{-1}$. Using the Simien \& Prugniel data, de Rijcke et al. (2006) constructed dynamical models for all three Local Group dEs, concluding that, within the half-light radii, all three galaxies have mass-to-light ratios above that expected from the stellar populations alone.

In this paper, we present accurate radial velocities out to $8 r_{\text {eff }}$ in the Local Group dE galaxies NGC 147 and NGC 185, based on Keck/DEIMOS multi-slit spectroscopy for 520 and 442 red giant branch (RGB) stars, respectively. The paper is organized as follows: in Section 2, we discuss target selection for our DEIMOS slitmasks, the observing procedure, and data reduction. In Section 3, we discuss the velocity, velocity dispersion, and metallicity profiles. In Section 4, we construct two-integral dynamical models for these galaxies and discuss the best-fit mass distribution and dark matter content. Finally, in Section 5, we discuss the implications of these results for the formation of $\mathrm{dE}$ galaxies.

Throughout this paper, we adopt distance moduli determined by McConnachie et al. (2005) via the tip of the RGB method. The distance modulus for NGC 147 is $(m-M)_{0}=24.43 \pm 0.04$ $(675 \pm 27 \mathrm{kpc})$ and NGC 185 is $(m-M)_{0}=24.23 \pm 0.03$ $(616 \pm 26 \mathrm{kpc})$. This places NGC 147 and NGC 185 at projected distances of 140 and $185 \mathrm{kpc}$ from their parent galaxy M 31, respectively.

\section{DATA}

\subsection{Target Selection}

Individual stars in NGC 147 and NGC 185 were targeted for spectroscopy according to their probability of being a RGB star based on Canada-France-Hawaii Telescope (CFHT) CFH12K mosaic imaging in the $R$ and $I$ bands (Battinelli \& Demers 2004a, $2004 b$ ). The CCD mosaic covers a $42^{\prime} \times 28^{\prime}$ region centered on each galaxy with 0'.206 pixels. Stellar photometry from these images extends 2 mag below the tip of the RGB. The spatial distribution of stars is shown in Figure 1. Color-magnitude diagrams are shown in Figure 2.

The absolute magnitude of the RGB for a metal-poor stellar population is $M_{I} \sim-4$. At the assumed distance and reddening of NGC 147 and NGC 185, the apparent magnitude of the RGB population is $I_{o} \sim 20.3$ (see Figure 2). Since internal 

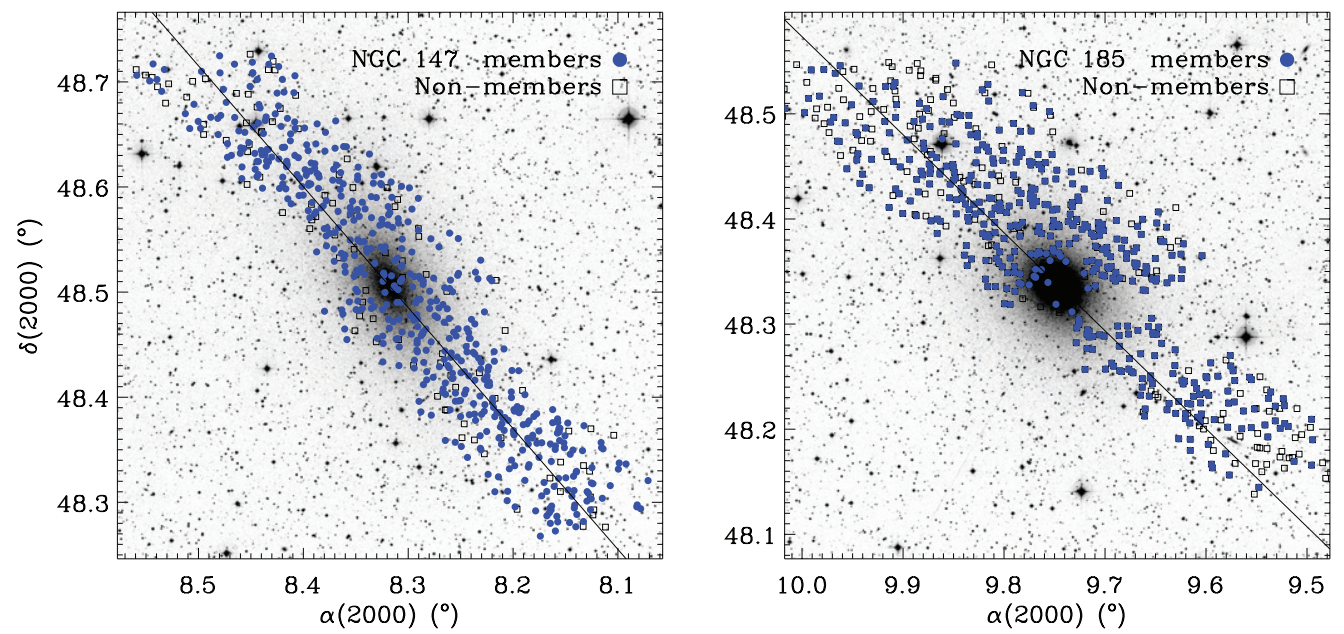

Figure 1. Palomar Sky Survey images of the two Local Group dE galaxies, NGC 147 (left) and NGC 185 (right). Images are $30^{\prime} \times 30^{\prime}$; north is up, east is to the left. The major axis of each galaxy is indicated by the solid line. The spatial position of Keck/DEIMOS spectroscopically confirmed member stars are shown as blue circles, non-members are plotted as open squares.

(A color version of this figure is available in the online journal.)
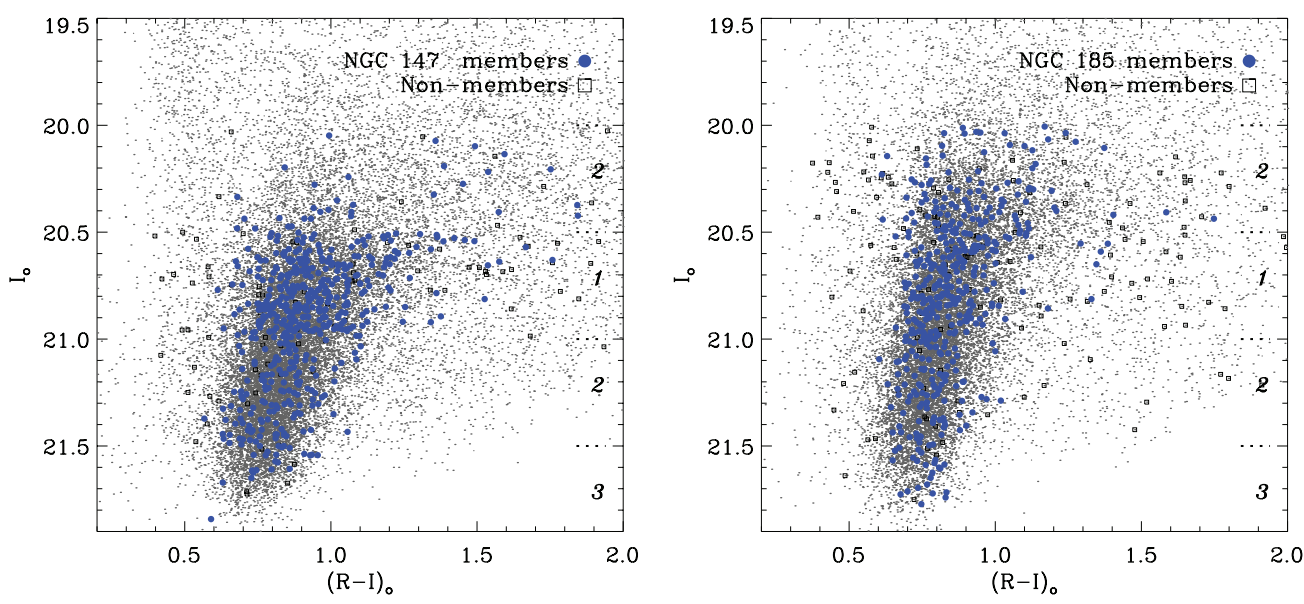

Figure 2. Color-magnitude diagrams for NGC 147 (left) and NGC 185 (right) based on Battinelli \& Demers (2004a, 2004b) $R$ - and $I$-band CHFT12K photometry, corrected for extinction, in a $42^{\prime} \times 28^{\prime}$ region centered on each galaxy. Blue circles are Keck/DEIMOS spectroscopically confirmed members of each galaxy, open squares are non-members. The numbered regions indicate the location of our primary (1), secondary (2), and tertiary (3) spectroscopic priorities.

(A color version of this figure is available in the online journal.)

metallicity variations will cause a spread in the colors of RGB stars, we select spectroscopic targets based primarily on apparent magnitude rather than color. We note that there is a 0.1 0.2 mag photometric zero-point error between individual CCD chips in the CFHT photometry which we discovered after our spectroscopic observations were completed. While this led us to use an alternative data set to determine the surface brightness profiles in Section 4.1, the error had little to no effect on our targeting efficiencies.

For both dEs, highest priority in the spectroscopic target list was assigned to stars between $20.5 \leqslant I_{o} \leqslant 21.0$. Second priority was given to stars between $20.0 \leqslant I_{o}<20.5$ and $21.0<I_{o} \leqslant 21.5$; lowest priority was assigned to objects $I_{o}>21.5$. To minimize Galactic foreground contamination, targets were required to have $(R-I)_{o}>0.2$. Stars with photometric errors larger than $\sigma_{I}>0.1$ were rejected, as were stars having neighbors of equal or greater brightness within a radius $r<4^{\prime \prime}$. The photometric criteria used for spectroscopic target selection are shown in Figure 2.

Slitmasks were created using the DEIMOS dsimulator slitmask design software. For each slitmask, the software fills in the mask area to the extent possible with the highest priority input targets. It then fills in the remaining space on the slitmask with lower priority targets. The specifics of each mask are listed in Table 2.

\subsection{Observations and Data Reduction}

The data were taken with the Keck II $10 \mathrm{~m}$ telescope and the DEIMOS spectrograph (Faber et al. 2003). Four multislit masks were observed in NGC 147 on the nights of 2005 August 28 and September 5-7. Five masks were observed in NGC 185 on 2006 September 16. Mask positions, exposure times, and other observing details are given in Table 2. The masks were observed with the 1200 line $\mathrm{mm}^{-1}$ grating covering a wavelength region $6400-9100 \AA$. The spectral dispersion of this setup is $0.33 \AA$, and the resulting spectral resolution, taking into account the anamorphic distortion, is $1.37 \AA$ (FWHM, equivalent to $47 \mathrm{~km} \mathrm{~s}^{-1}$ at the Ca II triplet). The spatial scale is $0 ! 12$ pixel $^{-1}$ and slitlets were 0 ! 7 wide. The minimum slit length was $4^{\prime \prime}$ which allows adequate sky subtraction; the minimum spatial separation between slit ends was 0".4 (three pixels). 
Table 2

Keck/DEIMOS Multi-slitmask Observing Parameters

\begin{tabular}{cccrccc}
\hline \hline $\begin{array}{c}\text { Mask } \\
\text { Name }\end{array}$ & $\begin{array}{c}\alpha(\mathrm{J} 2000) \\
(\mathrm{h}: \mathrm{m}: \mathrm{s})\end{array}$ & $\begin{array}{c}\delta(\mathrm{J} 2000) \\
\left({ }^{\circ}:^{\prime}:^{\prime \prime}\right)\end{array}$ & $\begin{array}{c}\text { P.A. } \\
(\mathrm{deg})\end{array}$ & $\begin{array}{c}t_{\exp } \\
(\mathrm{sec})\end{array}$ & No. of Slits & $\begin{array}{c}\% \text { Useful } \\
\text { Spectra }\end{array}$ \\
\hline N147_1 & $0: 32: 54.2$ & $48: 22: 48.0$ & 34 & 3300 & 190 & $87 \%$ \\
N147_2 & $0: 32: 56.6$ & $48: 22: 48.0$ & 34 & 3600 & 184 & $79 \%$ \\
N147_3 & $0: 33: 35.0$ & $48: 37: 09.5$ & 34 & 3600 & 195 & $72 \%$ \\
N147_4 & $0: 33: 36.0$ & $48: 37: 09.5$ & 214 & 3600 & 183 & $82 \%$ \\
N185_1 & $0: 39: 36.0$ & $48: 26: 06.0$ & 41 & 3240 & 155 & $92 \%$ \\
N185_2 & $0: 39: 14.4$ & $48: 26: 24.0$ & 221 & 2040 & 143 & $78 \%$ \\
N185_3 & $0: 38: 39.5$ & $48: 14: 58.2$ & 41 & 3120 & 135 & $88 \%$ \\
N185_4 & $0: 38: 25.4$ & $48: 15: 42.1$ & 221 & 3060 & 139 & $42 \%$ \\
N185_5 & $0: 39: 03.6$ & $48: 26: 24.0$ & 41 & 3060 & 143 & $92 \%$ \\
\hline
\end{tabular}

Notes. Right ascension, declination, position angle, and total exposure time for each Keck/DEIMOS slitmask. The final two columns refer to the total number of slitlets on each mask and the percentage of those slitlets for which a redshift was measured. Note that the percentage of good slits for N185_4 is low due to a data reduction failure for four out of eight CCDs.

Spectra were reduced using a modified version of the spec $2 \mathrm{~d}$ software pipeline (version 1.1.4) developed by the DEEP2 team at the University of California, Berkeley for that survey. A detailed description of the two-dimensional reductions can be found in Simon \& Geha (2007). The final one-dimensional spectra are rebinned into logarithmic wavelength bins with $15 \mathrm{~km} \mathrm{~s}^{-1}$ pixel $^{-1}$. An example DEIMOS spectrum taken with the same settings is shown in Figure 4 of Geha et al. (2006).

\subsection{Radial Velocities and Error Estimates}

Radial velocities were measured by cross-correlating the observed science spectra with a series of high signal-to-noise stellar templates. Stellar templates were observed with Keck/ DEIMOS using the same setup as described in Section 2.2 and cover a wide range of stellar types (F8 to M8 giants, subgiants and dwarf stars) and metallicities $([\mathrm{Fe} / \mathrm{H}]=-2.12$ to +0.11$)$. We calculate and apply a telluric correction to each science spectrum by cross-correlating a hot stellar template with the night sky absorption lines following the method in Sohn et al. (2007). The telluric correction accounts for the velocity error due to miscentering the star within the 0 '.7 slit caused by small mask rotations or astrometric errors. We apply both a telluric and heliocentric correction to all velocities presented in this paper.

We determine the random component of our velocity errors using a Monte Carlo bootstrap method. Noise is added to each pixel in the one-dimensional science spectrum, we then recalculate the velocity and telluric correction for 1000 noise realizations. Error bars are defined as the square root of the variance in the recovered mean velocity in the Monte Carlo simulations. The systematic contribution to the velocity error was determined by Simon \& Geha (2007) to be $2.2 \mathrm{~km} \mathrm{~s}^{-1}$ based on repeated independent measurements of individual stars. The systematic error contribution is expected to be constant as the spectrograph setup and velocity cross-correlation routines are identical. We add the random and systematic errors in quadrature to arrive at the final velocity error for each science measurement. Radial velocities were successfully measured for 1227 of the 1483 extracted spectra across the nine observed DEIMOS masks. The majority of spectra for which we could not measure a redshift did not have sufficient signal to noise. The fitted velocities were visually inspected to ensure reliability.

\subsection{Membership Criteria}

The expected velocity distributions of NGC 147 and NGC 185 overlap with both the stellar velocity distribution of the M31 halo and the foreground Milky Way disk and halo. Thus, a simple velocity cut is not sufficient to define membership in these $\mathrm{dE}$ galaxies. We determine membership in NGC 147 and NGC 185 using a modified version of the probability method of Gilbert et al. (2006). This method computes a membership probability for each star based on the assumption that $\mathrm{dE}$ stars have different physical properties than the contaminating foreground and background populations.

The Gilbert et al. (2006) method computes the probability that a given star is either a RGB star at the distance of NGC 147 or NGC 185, or a foreground Milky Way dwarf star based on the star's location in four spectroscopic and photometric diagnostics: line of sight velocity, strength of the surfacegravity sensitive Na II absorption line at $\sim 8190 \AA$, position in a color-magnitude diagram, and a comparison of photometric (CMD-based) and spectroscopic (based on the Ca II triplet at $\sim 8500 \AA$ A) metallicity estimates. A star's position in a diagnostic is compared to probability distribution functions based on appropriate training sets to determine the likelihood that the star is a RGB or foreground dwarf star. The likelihoods from the individual diagnostics are then combined to produce an overall likelihood.

For the present analysis, several modifications were made to the method published in Gilbert et al. (2006), which was optimized to identify RGB stars in the halo of M31. Several of the diagnostics utilize Johnson-Cousins $V$ and $I$ magnitudes, while the $\mathrm{dE}$ photometry was taken in the $R$ and $I$ bands. The CMD diagnostic and spectroscopic versus photometric metallicity diagnostic were re-derived using VandenBerg et al. (2006) isochrones in $(I, R-I)$ shifted to the distance of each $\mathrm{dE}$. To utilize the Na II diagnostic, VandenBerg isochrones were used to define a relation between $R-I$ and $V-I$, which was then used to derive a $V-I$ color for each star. The velocity distribution of each $\mathrm{dE}$ is modeled as a Gaussian, with a constant velocity dispersion and a central velocity that varies with distance along the semi-major axis. We expect little contamination from M 31 halo stars at these radii ( $<1$ star per mask; Guhathakurta et al. 2005).

Stars that are more than 3 times more likely to be a RGB star at the distance of the $\mathrm{dE}$ than a foreground dwarf star, and which have a spatial position and line-of-sight velocity consistent with that of the $\mathrm{dE}$, are classified as members. Given these membership criteria, we identify 520 and 442 RGB stars as members of the dE galaxies NGC 147 and NGC 185, respectively. Velocities and velocities errors for individual member stars are listed in Tables 6 and 7 in the electronic version of the paper, or on request from the authors.

\section{RESULTS}

The measured velocities of individual stars allow us to probe the dynamics of NGC 147 and NGC 185 to much larger radius than possible via integrated-light spectroscopy. In order to compare to previous results, and to construct dynamical models, we bin the individual velocity measurements based on their major-axis distance $x$, using bin widths $\Delta x=2$ '.05 for NGC 147 and $\Delta x=1$ 1.87 for NGC 185. The bin widths were chosen to roughly match the effective radius of each $\mathrm{dE}$. The bin with integer index number $i$ spans values of $x$ from $\left(i-\frac{1}{2}\right) \Delta x$ to $\left(i+\frac{1}{2}\right) \Delta x$. The value of $x$ for each star was calculated 
Table 3

Observed and Model Quantities

\begin{tabular}{|c|c|c|c|c|}
\hline Row & Quantity & Units & NGC 147 & NGC 185 \\
\hline \multicolumn{5}{|c|}{ Photometric Properties } \\
\hline (1) & $m_{V, 0}$ & mag & $8.95 \pm 0.05$ & $8.57 \pm 0.05$ \\
\hline (2) & $(B-V)_{0}$ & mag & $0.76 \pm 0.07$ & $0.72 \pm 0.07$ \\
\hline (3) & $\mathrm{PA}_{\text {maj }}$ & degrees & 208.4 & 222.9 \\
\hline (4) & $q_{a}$ & & 0.56 & 0.77 \\
\hline (5) & $i$ & degrees & 77.5 & 63.7 \\
\hline (6) & $q_{t}$ & & 0.53 & 0.70 \\
\hline (7) & $n$ & & 1.04 & 1.76 \\
\hline (8) & $j_{0}\left(q_{t} / q_{a}\right)$ & $L_{\odot} \mathrm{pc}^{-3}$ & $1.074 \times 10^{-2}$ & $3.008 \times 10^{-2}$ \\
\hline (9) & $b$ & $\operatorname{arcmin}$ & 10.72 & 5.48 \\
\hline (10) & $\alpha$ & & -0.940 & -1.439 \\
\hline (11) & $\delta$ & & -3.982 & -2.038 \\
\hline (12) & $\Delta x$ & $\operatorname{arcmin}$ & 1.87 & 2.00 \\
\hline$(13)$ & $\Delta y$ & $\operatorname{arcmin}$ & 5.00 & 8.00 \\
\hline \multicolumn{5}{|c|}{ Dynamical Properties } \\
\hline (14) & $V_{\text {sys }}$ & $\mathrm{km} \mathrm{s}^{-1}$ & $-193.1 \pm 0.8$ & $-203.8 \pm 1.1$ \\
\hline (15) & $V_{\max , \mathrm{obs}}$ & $\mathrm{km} \mathrm{s}^{-1}$ & $17 \pm 2$ & $15 \pm 5$ \\
\hline (16) & $\langle\sigma\rangle$ & $\mathrm{km} \mathrm{s}^{-1}$ & $16 \pm 1$ & $24 \pm 1$ \\
\hline (17) & $\left\langle V_{\mathrm{rms}}\right\rangle$ & $\mathrm{km} \mathrm{s}^{-1}$ & $19.1 \pm 1.1$ & $24.6 \pm 1.2$ \\
\hline (18) & $k$ & & $0.74 \pm 0.06$ & $0.47 \pm 0.08$ \\
\hline (19) & $M / L_{V}$ & $M_{\odot} / L_{\odot, V}$ & $4.24 \pm 0.56$ & $4.57 \pm 0.56$ \\
\hline (20) & $M / L_{B}$ & $M_{\odot} / L_{\odot, B}$ & $4.71 \pm 0.69$ & $4.90 \pm 0.68$ \\
\hline$(21)$ & Mass & $M_{\odot}$ & $5.6 \times 10^{8}$ & $7.2 \times 10^{8}$ \\
\hline \multicolumn{5}{|c|}{ Metallicity } \\
\hline (22) & {$[\mathrm{Fe} / \mathrm{H}]$} & $\operatorname{dex}$ & $-1.1 \pm 0.1$ & $-1.3 \pm 0.1$ \\
\hline (23) & $\sigma_{[\mathrm{Fe} / \mathrm{H}]}$ & $\operatorname{dex}$ & 0.4 & 0.5 \\
\hline
\end{tabular}

Notes. Quantities for the two dE galaxies, with uncertainties included where relevant. Row 1 lists the observed total $m_{V, 0}$ magnitude from de Vaucouleurs et al. (1991; RC3), corrected for the foreground extinction listed in Table 1. Row 2 lists the extinction corrected color $(B-V)_{0}$ from RC3. Row 3 gives the adopted major-axis position angle, based on the outermost radius in Kent's (1987) photometry. Row 4 lists the apparent axial ratio of the models, which is the average axial ratio in the radial range $1^{\prime}-5^{\prime}$ in the data of Kent (1987). Row 5 lists the statistically most likely inclination of each galaxy, based on the observed axial ratio $q_{a}$ and the distribution of intrinsic axial ratios $q_{t}$ from Tremblay \& Merritt (1995). Row 6 lists the true axial ratio of each galaxy, based on the observed axial ratio $q_{a}$ and the listed inclination. Row 7 lists the value $n$ of the best-fitting Sérsic profile, which is shown in Figure 4. Rows 8-11 give the parameters of the best-fitting luminosity density parameterization $j(R, z)$ in Equation (1). Row 12 lists the width $\Delta x$ of the bins used in binning the stellar velocities along the major axis. Row 13 lists the approximate extent in the minor-axis direction of the region around the major axis over which data were obtained. Row 14 lists the heliocentric systemic velocity $V_{\text {sys }}$. Row 15 lists the weighted average value of $V_{\text {rms }}$ inside $4^{\prime}$. Row 16 lists the maximum observed rotational velocity, and Row 17 lists the averaged observed velocity dispersion. Rows 18-20 are the mass-to-light ratio $M / L$, and the rotation parameter $k$ (see Equation (2)) inferred by fitting the models to our new kinematical data. The listed uncertainties in $M / L$ include the propagated uncertainties in the galaxy distances $D$ and the photometric uncertainties in the surface brightness profiles and color transformations. The $B$-band mass-to-light ratios were obtained from the modeled $V$-band values using the galaxy color $(B-V)_{0}$ and the solar color $(B-V)_{\odot}=0.65$. The characteristic mass $M=(M / L) \times L$ of each galaxy is listed in Row 21. Row 22 lists the average metallicity as described in Section 3.2. Row (23) lists the internal metallicity dispersion in each $\mathrm{dE}$.

using spherical trigonometry and a zenithal projection, using the galaxy center positions and major axis position angles (Tables 1 and 3); positive radii lie on the northeast side of each galaxy.

We analyze our kinematic data following the procedure described in Appendix A of van der Marel \& Anderson (2009). For each bin number $i$, we calculated the weighted mean velocity $V_{i}$ of the individual stellar velocities, $v_{j}$, and its formal error $\Delta V_{i}$.
Individual weights were set equal to the quadrature sum of the observational velocity errors $\Delta v_{j}$ and the velocity dispersion $\sigma_{i}$ for the given bin. The latter was determined by finding the value that maximizes the likelihood of the set of velocity residuals $v_{j}-V_{i}$. The formal error $\Delta \sigma_{i}$ was calculated using a Monte Carlo approach, taking into account the observational velocity errors $\Delta v_{j}$. The systemic radial velocity, $V_{\text {sys }}$, for each galaxy was determined by finding the value that provides the lowest $\chi^{2}$ when the dynamical models described in Section 4 were fit to the data and is subtracted from each of the binned major-axis velocities $V_{i}$. The systemic velocities for NGC 147 and NGC 185 are $V_{\text {sys }}=-193.1 \pm 0.8 \mathrm{~km} \mathrm{~s}^{-1}$ and $-203.8 \pm 1.1 \mathrm{~km} \mathrm{~s}^{-1}$, respectively. This is consistent with literature values from Bender et al. (1991) and the velocity compilation from the NASA Extragalactic Database.

To further improve the signal-to-noise ratio, we folded the profiles above by taking the weighted average of bins on opposite sides of the galaxy (i.e., bins $i$ and $-i$ ), using the expected anti-symmetry and symmetry of the rotation and dispersion profiles, respectively. Values of the observed $V_{\text {rms }}$ and their formal errors were obtained by adding the results for $V$ and $\sigma$ in quadrature. The full kinematic profiles are shown in Figure 3 (also see Tables 4 and 5); the folded profiles are shown in Figure 5.

\subsection{The Velocity Profiles and Rotation in dE Galaxies}

We present the major-axis velocity profiles for our two $\mathrm{dEs}$ in Figure 3 . The profiles extend to slightly more than $14^{\prime}$ in each galaxy, equivalent to $7 r_{\text {eff }}$ and $9 r_{\text {eff }}$ in NGC 147 and NGC 185, respectively. Previous kinematic observations for each $\mathrm{dE}$ have been limited to within one $r_{\text {eff }}$ (Simien \& Prugniel 2002). In Figure 3, a clear rotation signal is seen even in the individual unbinned velocity distribution of stars. The binned major-axis velocity profiles in this figure show a well-defined rotation curve profile. The maximum observed rotational velocity is $17 \pm 2 \mathrm{~km} \mathrm{~s}^{-1}$ and $15 \pm 5 \mathrm{~km} \mathrm{~s}^{-1}$ for NGC 147 and NGC 185, respectively, determined from the maximum data point in the folded rotation curves (Figure 5). While our velocity profiles flatten at large radius, it is unlikely that, even at these large radial distances, we have reached the maximum rotation velocity in these galaxies. The velocity dispersion profiles in both dEs decline gently with radius. The weighted average values are $\sigma=16 \pm 1 \mathrm{~km} \mathrm{~s}^{-1}$ and $24 \pm 1 \mathrm{~km} \mathrm{~s}^{-1}$ for NGC 147 and NGC 185, respectively.

The significant rotation velocities we measure are in fact consistent with literature results of little to no rotation. Previous observations of NGC 147 and NGC 185 were limited to within $2^{\prime}$, corresponding to the inner three data points of Figure 3. Fitting a simple straight line to our inner data points, we predict a rotation velocity of merely $0.8 \mathrm{~km} \mathrm{~s}^{-1}$ at this radius for NGC 185, well below the observational errors of both Bender et al. (1991) and Simien \& Prugniel (2002), and fully consistent with their conclusion of no rotation. For NGC 147, we predict a rotation velocity of $4.3 \mathrm{~km} \mathrm{~s}^{-1}$ within $2^{\prime}$. This is marginally inconsistent with both Simien \& Prugniel (2002), who found no rotation at this radius, and Bender et al. (1991), who measured $6.5 \pm 1.1 \mathrm{~km} \mathrm{~s}^{-1}$, but is consistent with the average of these two observations. Our discovery of rotation at radii larger than $r_{\text {eff }}$ has significant implications for studies of more distant $\mathrm{dE}$ galaxies. We will discuss this issue further below and in Section 5.

Early kinematic observations of the Local Group dEs (Davies et al. 1983; Bender et al. 1991) established the paradigm that 
Table 4

Major-axis Profile of NGC 147

\begin{tabular}{|c|c|c|c|c|c|c|c|c|}
\hline $\begin{array}{c}x \\
(\operatorname{arcmin}) \\
\end{array}$ & $\begin{array}{r}\alpha(\mathrm{J} 2000) \\
(\mathrm{h}: \mathrm{m}: \mathrm{s}) \\
\end{array}$ & $\begin{array}{c}\delta(\mathrm{J} 2000) \\
\left({ }^{\circ}:^{\prime}:^{\prime \prime}\right) \\
\end{array}$ & $\begin{array}{c}V \\
\left(\mathrm{~km} \mathrm{~s}^{-1}\right) \\
\end{array}$ & $\begin{array}{c}V_{\text {err }} \\
\left(\mathrm{km} \mathrm{s}^{-1}\right)\end{array}$ & $\begin{array}{c}\sigma \\
\left(\mathrm{km} \mathrm{s}^{-1}\right) \\
\end{array}$ & $\begin{array}{c}\sigma_{\mathrm{err}} \\
\left(\mathrm{km} \mathrm{s}^{-1}\right)\end{array}$ & $\begin{array}{c}{[\mathrm{Fe} / \mathrm{H}]} \\
(\mathrm{dex})\end{array}$ & $\begin{array}{c}{[\mathrm{Fe} / \mathrm{H}]_{\mathrm{en}}} \\
(\mathrm{dex})\end{array}$ \\
\hline-14.7 & $00: 32: 34.5$ & $+48: 19: 25.3$ & -15.4 & 3.3 & 14.2 & 2.4 & -0.7 & 0.1 \\
\hline-13.2 & $00: 32: 38.6$ & $+48: 20: 35.5$ & -18.4 & 3.2 & 16.4 & 2.3 & -0.9 & 0.1 \\
\hline-11.1 & $00: 32: 44.0$ & $+48: 22: 08.8$ & -16.4 & 3.2 & 16.7 & 2.3 & -0.7 & 0.1 \\
\hline-9.4 & $00: 32: 48.6$ & $+48: 23: 27.6$ & -11.0 & 3.5 & 21.5 & 2.5 & -0.8 & 0.1 \\
\hline-7.5 & $00: 32: 53.4$ & $+48: 24: 50.4$ & -11.5 & 3.4 & 18.0 & 2.5 & -0.9 & 0.1 \\
\hline-5.6 & $00: 32: 58.4$ & $+48: 26: 17.2$ & -10.0 & 2.7 & 15.5 & 2.0 & -0.7 & 0.1 \\
\hline-3.7 & $00: 33: 03.5$ & $+48: 27: 44.3$ & -6.8 & 3.8 & 21.3 & 2.8 & -1.0 & 0.1 \\
\hline-1.9 & $00: 33: 08.3$ & $+48: 29: 07.1$ & -2.6 & 3.6 & 21.1 & 2.7 & -1.1 & 0.2 \\
\hline-0.1 & $00: 33: 12.8$ & $+48: 30: 25.9$ & -1.8 & 2.8 & 17.2 & 2.1 & -0.8 & 0.2 \\
\hline 1.9 & $00: 33: 18.1$ & $+48: 31: 57.0$ & 1.9 & 3.7 & 18.3 & 2.9 & -1.0 & 0.2 \\
\hline 3.7 & $00: 33: 22.9$ & $+48: 33: 19.8$ & 8.7 & 2.9 & 16.2 & 2.2 & -0.6 & 0.1 \\
\hline 5.6 & $00: 33: 27.8$ & $+48: 34: 44.8$ & 12.9 & 3.7 & 20.5 & 2.7 & -1.1 & 0.1 \\
\hline 7.4 & $00: 33: 32.7$ & $+48: 36: 09.4$ & 11.8 & 2.8 & 15.6 & 2.1 & -0.9 & 0.1 \\
\hline 9.2 & $00: 33: 37.4$ & $+48: 37: 30.4$ & 14.8 & 2.8 & 16.4 & 2.1 & -0.9 & 0.1 \\
\hline 11.3 & $00: 33: 42.7$ & $+48: 39: 01.4$ & 13.8 & 2.5 & 10.1 & 1.9 & -1.2 & 0.2 \\
\hline 13.1 & $00: 33: 47.5$ & $+48: 40: 24.2$ & 16.3 & 2.8 & 9.2 & 2.2 & -1.3 & 0.2 \\
\hline 14.7 & $00: 33: 51.8$ & $+48: 41: 38.8$ & 17.2 & 3.5 & 7.7 & 3.2 & -0.8 & 0.1 \\
\hline
\end{tabular}

Notes. The velocity $(V)$, velocity dispersion $(\sigma)$, and metallicity $([\mathrm{Fe} / \mathrm{H}])$ as a function of the binned major-axis distance $x$ in arcminutes away from the galaxy center. The right ascension and declination are given for each radial bin: positive radial bins on the northeast side of the galaxy, negative radial bins are on the southwest.

Table 5

Major-axis Profile of NGC 185

\begin{tabular}{|c|c|c|c|c|c|c|c|c|}
\hline $\begin{array}{c}x \\
(\operatorname{arcmin}) \\
\end{array}$ & $\begin{array}{l}\alpha(\mathrm{J} 2000) \\
(\mathrm{h}: \mathrm{m}: \mathrm{s})\end{array}$ & $\begin{array}{c}\delta(\mathrm{J} 2000) \\
\left({ }^{\circ}:^{\prime}::^{\prime \prime}\right)\end{array}$ & $\begin{array}{c}V \\
\left(\mathrm{~km} \mathrm{~s}^{-1}\right) \\
\end{array}$ & $\begin{array}{c}V_{\text {err }} \\
\left(\mathrm{km} \mathrm{s}^{-1}\right)\end{array}$ & $\begin{array}{c}\sigma \\
\left(\mathrm{km} \mathrm{s}^{-1}\right) \\
\end{array}$ & $\begin{array}{c}\sigma_{\text {err }} \\
\left(\mathrm{km} \mathrm{s}^{-1}\right) \\
\end{array}$ & $\begin{array}{c}{[\mathrm{Fe} / \mathrm{H}]} \\
(\mathrm{dex})\end{array}$ & $\begin{array}{c}{[\mathrm{Fe} / \mathrm{H}]_{\mathrm{err}}} \\
(\mathrm{dex})\end{array}$ \\
\hline-12.3 & $00: 38: 22.2$ & $+48: 11: 53.5$ & -12.6 & 9.1 & 28.8 & 6.5 & -1.4 & 0.1 \\
\hline-9.9 & $00: 38: 29.0$ & $+48: 13: 30.0$ & -9.3 & 5.4 & 24.0 & 3.9 & -1.4 & 0.1 \\
\hline-8.1 & $00: 38: 34.5$ & $+48: 14: 46.0$ & -7.0 & 3.6 & 19.6 & 2.6 & -0.8 & 0.1 \\
\hline-5.9 & $00: 38: 40.6$ & $+48: 16: 10.9$ & -3.6 & 6.7 & 27.6 & 4.8 & -1.2 & 0.2 \\
\hline-4.0 & $00: 38: 46.2$ & $+48: 17: 29.4$ & -7.9 & 6.8 & 31.8 & 4.9 & -0.9 & 0.3 \\
\hline-2.0 & $00: 38: 52.1$ & $+48: 18: 52.2$ & -6.1 & 4.5 & 26.5 & 3.2 & -0.7 & 0.3 \\
\hline-0.1 & $00: 38: 57.8$ & $+48: 20: 12.8$ & 0.5 & 3.8 & 23.9 & 2.7 & -0.8 & 0.2 \\
\hline 1.9 & 00:39:03.6 & $+48: 21: 33.5$ & -0.3 & 3.1 & 23.0 & 2.2 & -0.9 & 0.1 \\
\hline 4.0 & 00:39:09.7 & $+48: 22: 58.4$ & 3.3 & 3.3 & 23.6 & 2.4 & -0.9 & 0.1 \\
\hline 6.0 & $00: 39: 15.6$ & $+48: 24: 21.2$ & 7.4 & 3.2 & 20.5 & 2.3 & -1.4 & 0.1 \\
\hline 8.0 & $00: 39: 21.4$ & $+48: 25: 41.9$ & 5.8 & 3.3 & 20.9 & 2.4 & -1.3 & 0.1 \\
\hline 10.0 & $00: 39: 27.1$ & $+48: 27: 02.2$ & 11.7 & 3.9 & 20.0 & 2.8 & -1.4 & 0.1 \\
\hline 11.9 & $00: 39: 32.9$ & $+48: 28: 22.8$ & 11.6 & 4.7 & 18.8 & 3.5 & -1.5 & 0.1 \\
\hline 14.4 & $00: 39: 40.2$ & $+48: 30: 05.8$ & 10.5 & 6.1 & 22.0 & 4.4 & -1.5 & 0.1 \\
\hline
\end{tabular}

Note. See comments for Table 4.

$\mathrm{dE}$ galaxies are supported by anisotropic velocity dispersions, in contrast to low-luminosity elliptical galaxies which are rotationally supported. Our observations show that the Local Group dEs do indeed have significant rotational support. To demonstrate this, we compare the ratio of the observed and predicted values of the maximum rotational velocity to the average velocity dispersion using the quantity $\left(V_{\max } / \sigma\right)^{*}$. For a rotationally supported oblate galaxy, $\left(V_{\max } / \sigma\right)^{*}=1$; values less than unity imply anisotropic support. Using the measured ellipticity of each $\mathrm{dE}$ (Table 1), the measured maximum rotation velocity and the average velocity dispersion values from above, we compute $\left(V_{\max } / \sigma\right)^{*}=0.95$ and 0.91 for NGC 147 and NGC 185, respectively. Because our observed maximum rotational velocities are lower limits on the true maximum rotation, these $\left(V_{\max } / \sigma\right)^{*}$ values are also lower limits to the true values. For the third $\mathrm{dE}$ in the Local Group, NGC 205, we measured $\left(V_{\max } / \sigma\right)^{*}=0.3$ in Geha et al. (2006). The original value for NGC 205 may have been somewhat larger given that the outer parts of this galaxy have been tidal disrupted by interactions with $\mathrm{M} 31$. Thus, while the majority of kinematic support for the observed shapes of the Local Group dE galaxies comes from rotation, some anisotropic velocity dispersions are needed to fully explain the observed shapes. This is consistent with our dynamical modeling results in Section 4.3.

\subsection{Metallicity}

We measure the spectroscopic metallicities $([\mathrm{Fe} / \mathrm{H}])$ of individual RGB stars based on the Ca II triplet absorption lines near $\lambda=8500 \AA$. This method relies on an empirical calibration to Galactic globular clusters and is reliable for metallicities above $[\mathrm{Fe} / \mathrm{H}]>-2.5$ (Kirby et al. 2008), which is the case for our member stars. We calculate the equivalent widths (EWs) of the three Ca II absorption lines using the line and continuum definitions of Rutledge et al. (1997a) and convert this to [Fe/H] using the empirical calibration relationship of Rutledge et al. (1997b). 

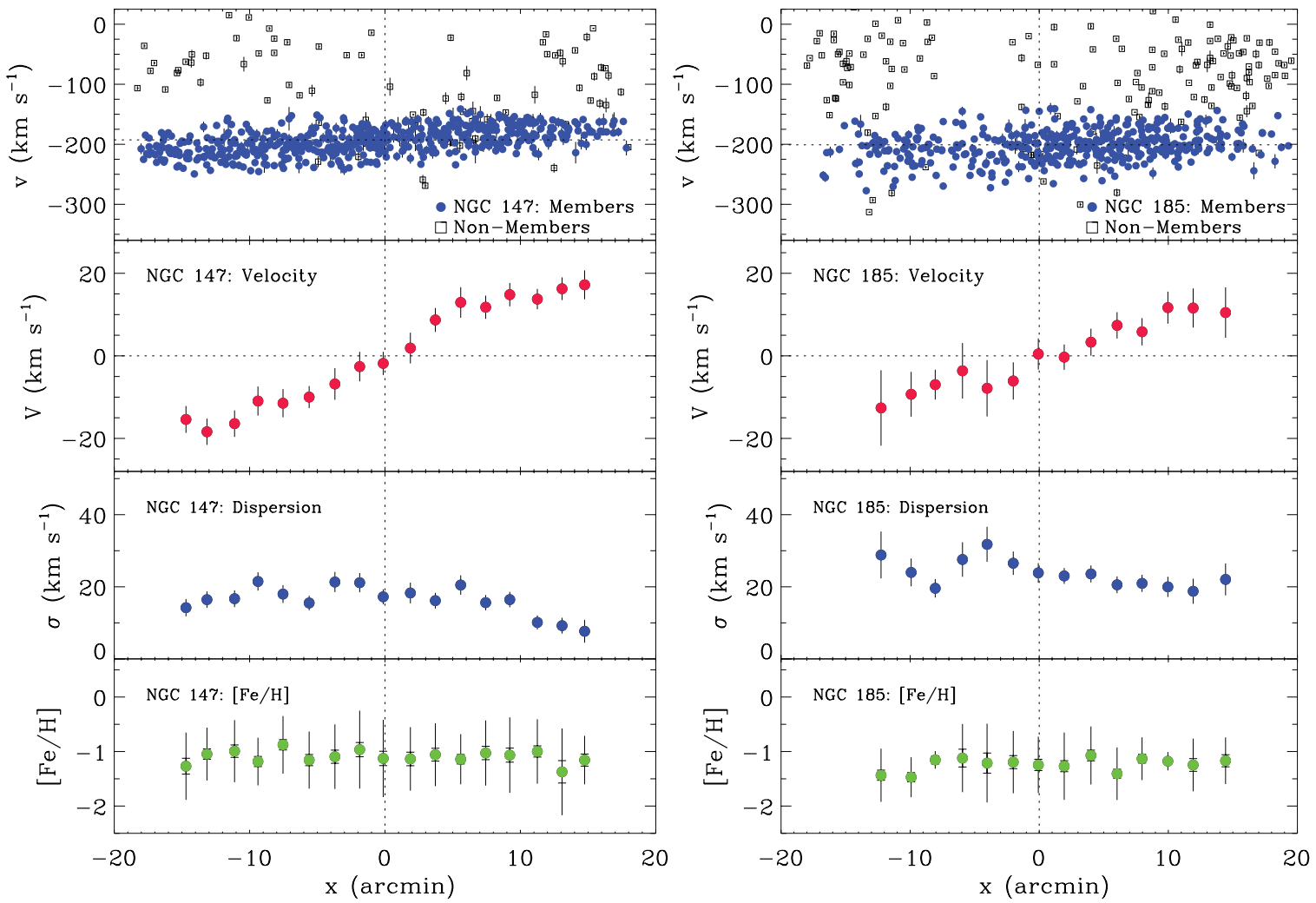

Figure 3. Top: individual velocities as a function of semi-major axis distance for $\mathrm{dE}$ member stars (blue circles) and non-members (open squares) of NGC 147 (left) and NGC 185 (right). Positive radii correspond to the northeast side of each dE. Top middle: the binned major-axis velocity profile showing significant rotation velocities in each $\mathrm{dE}$ galaxy. Bottom middle: the velocity dispersion profiles are nearly flat to the last measured radial point. Bottom: the $[\mathrm{Fe} / \mathrm{H}] \mathrm{spectroscopic}$ metallicity as a function of semi-major axis distance. Barred errors indicate the error on the mean metallicity, non-barred errors indicate the metallicity dispersion in each bin.

(A color version of this figure is available in the online journal.)

Table 6

Keck/DEIMOS Velocity Measurements for NGC 147

\begin{tabular}{cccccccc}
\hline \hline$i$ & Name & $\begin{array}{c}\alpha(\mathrm{J} 2000) \\
(\mathrm{h} \mathrm{m} \mathrm{s})\end{array}$ & $\begin{array}{c}\delta(\mathrm{J} 2000) \\
\left({ }^{\prime} \prime \prime\right)\end{array}$ & $\begin{array}{c}I-\mathrm{mag} \\
(\mathrm{mag})\end{array}$ & $\begin{array}{c}(R-I) \\
(\mathrm{mag})\end{array}$ & $\begin{array}{c}v \\
\left(\mathrm{~km} \mathrm{~s}^{-1}\right)\end{array}$ & $\begin{array}{c}v_{\text {err }} \\
\left(\mathrm{km} \mathrm{s}^{-1}\right)\end{array}$ \\
\hline 1 & 11231 & $00: 33: 02.4$ & $+48: 23: 16.7$ & 21.3 & 0.97 & -235.5 & 3.3 \\
2 & 4456 & $00: 32: 23.9$ & $+48: 19: 53.8$ & 20.5 & 1.09 & -235.5 & 3.3 \\
3 & 4608 & $00: 32: 25.8$ & $+48: 18: 57.2$ & 20.7 & 1.14 & -206.2 & 2.8 \\
4 & 4778 & $00: 32: 27.6$ & $+48: 18: 13.8$ & 20.9 & 0.76 & -203.4 & 4.9 \\
5 & 4894 & $00: 32: 29.7$ & $+48: 19: 03.8$ & 21.1 & 0.94 & -209.9 & 3.2 \\
$\ldots$ & $\ldots$ & $\ldots$ & $\cdots$ & $\cdots$ & $\cdots$ & $\cdots$ & $\cdots$ \\
\hline
\end{tabular}

Notes. Velocity measurements for member stars of NGC 147. Position, apparent $I$-band magnitude, $(R-I)$ color, heliocentric radial velocity $(v)$, and velocity error $\left(v_{\text {err }}\right)$ for each star as determined in Section 2.3.

(This table is available in its entirety in a machine-readable form in the online journal. A portion is shown here for guidance regarding its form and content.).

We determine the error on the $[\mathrm{Fe} / \mathrm{H}]$ values with the Monte Carlo method described in Section 2.3, and add in quadrature a systematic uncertainty of $0.3 \AA$ as determined in Simon \& Geha (2007). We restrict this analysis to stellar spectra with signal-to-noise ratio greater than or equal to $5 \mathrm{pixel}^{-1}$.

The binned metallicity profiles for NGC 147 and NGC 185 are shown in the bottom panels of Figure 3. For NGC 147 and NGC 185, we find average metallicities of $[\mathrm{Fe} / \mathrm{H}]=-1.1 \pm 0.1$ and $[\mathrm{Fe} / \mathrm{H}]=-1.3 \pm 0.1$, respectively. This is in agreement with previous measurements from a variety of sources, including photometric and spectroscopic metallicity estimates (Han et al. 1997; Martínez-Delgado et al. 1999; Davidge 2005; Gonçalves et al. 2007). While the mean metallicity is well determined, there
Table 7

Keck/DEIMOS Velocity Measurements for NGC 185

\begin{tabular}{cccccccc}
\hline \hline$i$ & Name & $\begin{array}{c}\alpha(\mathrm{J} 2000) \\
(\mathrm{h}: \mathrm{m}: \mathrm{s})\end{array}$ & $\begin{array}{c}\delta(\mathrm{J} 2000) \\
\left({ }^{\prime}{ }^{\prime \prime}\right)\end{array}$ & $\begin{array}{c}I \text {-mag } \\
(\mathrm{mag})\end{array}$ & $\begin{array}{c}(R-I) \\
(\mathrm{mag})\end{array}$ & $\begin{array}{c}v \\
\left(\mathrm{~km} \mathrm{~s}^{-1}\right)\end{array}$ & $\begin{array}{c}v_{\text {err }} \\
\left(\mathrm{km} \mathrm{s}^{-1}\right)\end{array}$ \\
\hline 1 & 11396 & $00: 38: 58.6$ & $+48: 21: 52.3$ & 20.5 & 0.66 & -203.3 & 3.8 \\
2 & 11487 & $00: 38: 58.9$ & $+48: 23: 33.3$ & 20.9 & 1.18 & -202.3 & 4.4 \\
3 & 11515 & $00: 38: 59.0$ & $+48: 22: 03.4$ & 20.8 & 1.01 & -190.0 & 5.9 \\
4 & 11522 & $00: 38: 59.0$ & $+48: 22: 34.5$ & 21.0 & 0.69 & -215.6 & 4.1 \\
5 & 12017 & $00: 39: 00.2$ & $+48: 22: 09.0$ & 20.1 & 0.96 & -175.7 & 2.8 \\
$\cdots$ & $\cdots$ & $\cdots$ & $\cdots$ & $\cdots$ & $\cdots$ & $\cdots$ & $\cdots$ \\
\hline
\end{tabular}

Note. Same as Table 6, but for NGC 185 .

(This table is available in its entirety in a machine-readable form in the online journal. A portion is shown here for guidance regarding its form and content.)

is significant internal metallicity dispersion of $0.5 \mathrm{dex}$ in each galaxy, assuming a Gaussian metallicity distribution function. Although the metallicity distribution function is asymmetric, given our errors the assumption of a Gaussian distribution is justified. In Figure 3, we plot both the error on the mean (barred error bars) in each radial bin and the internal metallicity dispersion (unbarred errors). Despite our significant radial coverage, we do not find evidence for radial metallicity gradients.

\section{DYNAMICAL MODELING}

We have constructed a set of dynamical models to provide an initial interpretation of the data. The software was developed by van der Marel et al. (1994) for modeling the Local Group galaxy M32 and used more recently in van der Marel \& van 
NGC 147
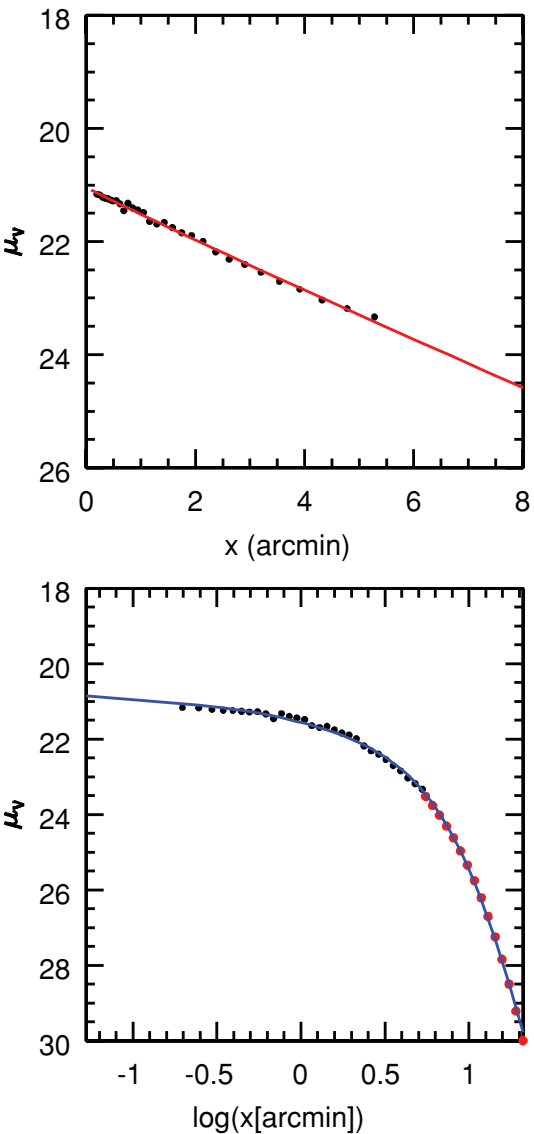

NGC 185
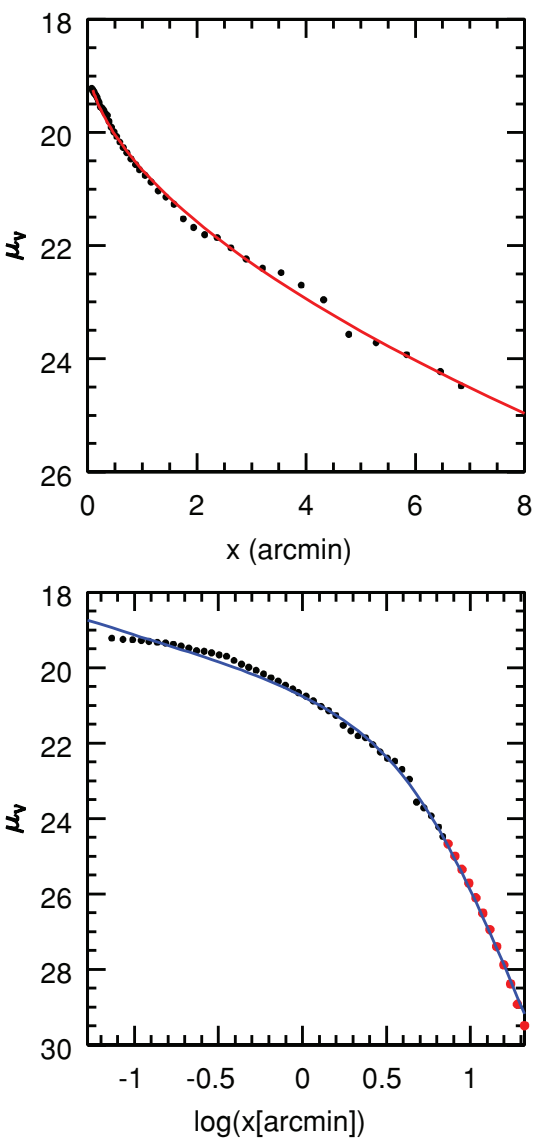

Figure 4. Extinction-corrected $V$-band surface brightness profiles in mag $\operatorname{arcsec}^{-2}$ of NGC 147 (left) and NGC 185 (right). Black dots show the data from Kent (1987), transformed to the $V$ band. Data inside of 1.5 times the seeing FWHM were ignored. The top panels have the linear major-axis radius $x$ along the abscissa, while the bottom panels have $\log (x)$ along the abscissa. Red curves in the top panels are the best Sérsic-profile fits to the data. Red dots in the bottom panels show the Sérsic extrapolation of the data to larger radii. Blue curves in the bottom panels are the predictions of the best-fitting luminosity density parameterization $j(R, z)$ in Equation (1). Parameters of the fits are listed in Table 3.

(A color version of this figure is available in the online journal.)

Dokkum (2007a). The models assume a two-integral form of the distribution function and are used to fit the binned profiles in Figure 5. Binning results some information loss and methods do exist to model unbinned data (e.g. Strigari et al. 2008; Chanamé et al. 2008; Coccato et al. 2009); however, we chose to bin the kinematic data here primarily to facilitate comparisons to previous work on these galaxies. A full analysis of this data set should preferably avoid spatial binning of the data, restrictive use of only the lowest order velocity moments, and allow for a more general form of the distribution function. We intend to construct such models in a future paper using the discrete Schwarzschild method of Chanamé et al. (2008).

Our present models are based on solutions of the Jeans equations of hydrostatic equilibrium with the following assumptions: (1) an oblate axisymmetric geometry with a constant axial ratio, (2) a constant mass-to-light ratio $M / L$, and (3) a two-integral distribution function of the form $f=f\left(E, L_{z}\right)$, where $E$ is the energy and $L_{z}$ is the angular momentum around the symmetry axis. Two-integral models have $\overline{v_{R}^{2}} \equiv \overline{v_{z}^{2}}$, so they can be thought of as the axisymmetric generalization of spherical isotropic models. A summary of the equations on which the models are based is provided in Section 2 of van der Marel $\&$ van Dokkum (2007a). We do not repeat this here, but focus instead on the specific application to NGC 147 and NGC 185.
Quantities for these galaxies that are relevant to the models are listed in Table 3 and are discussed below.

\subsection{Brightness and Density Profiles}

The modeling starts with the observed surface photometry of each galaxy. While the CFHT data discussed in Section 2.1 are appropriate for measuring surface brightness profiles, an unrecoverable calibration error resulted in a significant zeropoint error between CCDs. Instead, we use the data presented by Kent (1987) in the Gunn $r$ band. We transformed the Kent major-axis data to the Johnson $V$ band using the $(B-V)$ colors from the RC3 (see Table 3) combined with the transformation equation $V-r=0.486(B-V)-0.273$ (Jorgensen 1994). The resulting surface brightness profiles, corrected for extinction (Schlegel et al. 1998), are shown in Figure 4 and extend to 5.3 and 6.8 for NGC 147 and NGC 185, respectively. For both galaxies, this is less than half the extent of our kinematical data. To extrapolate the photometry to larger radii, we determined the best-fitting Sérsic profiles, which are overplotted in the top panels of Figure 4. The profiles fit the data well, with RMS residuals of 0.04 and 0.08 mag for NGC 147 and NGC 185, respectively.

We set the apparent axial ratio $q_{a}\left(q_{a}=1-\epsilon\right)$ equal to the average of the observed axial ratios in the radial range $1^{\prime}-5^{\prime}$ (see 

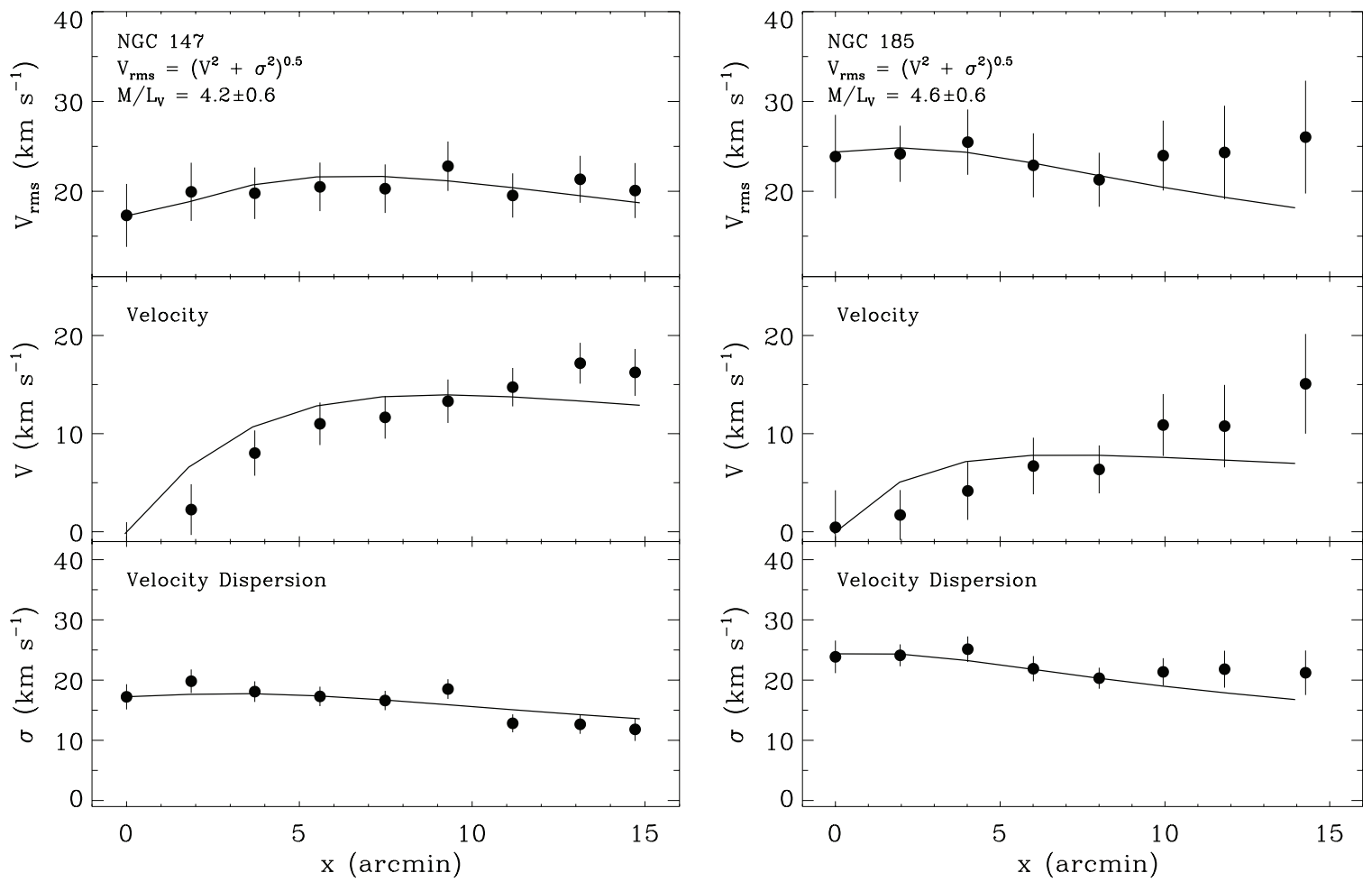

Figure 5. Major-axis kinematics of NGC 147 (left) and NGC 185 (right). Black dots show the binned results extracted from our data as described in the text. Black curves show the predictions of the best-fitting $f\left(E, L_{z}\right)$ models. Parameters of the fits are listed in Table 3 . The top panels provide evidence for an $M / L$ increase with radius in NGC 185, but not in NGC 147.

Table 3). Any systematic variations in the axial ratio over this radial range are small in both galaxies $(\lesssim 0.1 ;$ Kent 1987$)$. The total apparent magnitude, $V_{0}$, calculated using this axial ratio and our best-fitting Sérsic model above agree with that in the $\mathrm{RC} 3$ catalog to within $\sim 0.1-0.2$ mag for each $\mathrm{dE}$ galaxy. This provides added confidence in the use of Sérsic extrapolations at large radii.

The inclination of each $\mathrm{dE}$ is not well constrained by the data; however, both the predicted kinematical profiles and the inferred $M / L$ are generally insensitive to the assumed inclination (e.g. van der Marel \& van Dokkum 2007a, 2007b). The distribution of intrinsic axial ratios $q_{t}$ for elliptical galaxies is known (Tremblay \& Merritt 1995), and we assume this distribution for our dEs. We use this distribution as in van der Marel \& van Dokkum (2007a) to calculate the statistically most likely inclination. Since NGC 147 appears flatter on the sky than NGC 185, its most likely inclination is closer to edgeon $\left(i=78^{\circ}\right)$ than that for NGC $185\left(i=64^{\circ}\right)$. The intrinsic axial ratios $q_{t}$ corresponding to these assumed inclinations are given in Table 3. We discuss the dependence on inclination in Section 4.4.

We parameterize the three-dimensional luminosity density as

$$
j(R, z)=j_{0}\left(\frac{m}{b}\right)^{\alpha}\left[1+\left(\frac{m}{b}\right)^{2}\right]^{\delta}, \quad m^{2} \equiv R^{2}+z^{2} q_{t}^{-2} .
$$

Here $z$ is the symmetry axis of the galaxy and $(R, \phi, z)$ are the usual cylindrical coordinates. We fit the projected surface brightness (obtained by numerical line-of-sight integration of $j$ ) to the combination of the Kent (1987) data and its Sérsic-profile extrapolation to $20^{\prime}$. The surface brightness profile fits are shown in the bottom panels of Figure 4, and the model parameters are listed in Table 3. These results are independent of the assumed inclination, although $j_{0}$ depends on inclination through the ratio $\left(q_{t} / q_{a}\right)$, as well as on the adopted distances. The surface brightness models fit the extrapolated profiles adequately, with RMS residuals out to $20^{\prime}$ of 0.06 and 0.10 mag for NGC 147 and NGC 185 , respectively.

\subsection{Dynamics}

Given the $j(R, z)$ luminosity density, we assume a constant $M / L$ to calculate the gravitational potential. We then solve the Jeans equations to determine the intrinsic dynamical quantities, followed by a luminosity-weighted line-of-sight projection to calculate observable quantities. In particular, the models yield the quantity $V_{\mathrm{rms}} \equiv\left[V^{2}+\sigma^{2}\right]^{1 / 2}$, where $V$ is the mean velocity and $\sigma$ is the velocity dispersion. The quantity $V_{\text {rms }}$ scales linearly with $(M / L)^{1 / 2}$, but its spatial and radial dependences are otherwise uniquely determined. The quantities $V$ and $\sigma$ can also be predicted separately, but this requires the introduction of a free parameter (or function) $k$ that specifies how the second azimuthal velocity moment separates into mean and random components:

$$
\overline{v_{\phi}}=k\left[\overline{v_{\phi}^{2}}-\overline{v_{R}^{2}}\right]^{1 / 2} \text {. }
$$

For $k=0$, the model is non-rotating, whereas for $|k|=1$ the velocity dispersion tensor is isotropic and the model is a so-called "oblate isotropic rotator." The quantity $k$ is therefore similar to the quantity $(V / \sigma)^{*}$ discussed is Section 3.1, with the difference that $k$ is defined locally, while $(V / \sigma)^{*}$ is defined in terms of globally averaged projected quantities.

\subsection{Data-Model Comparison}

For proper comparison to the data, the model predictions were binned on the sky in rectangles of size $\Delta x \times \Delta y$. Here $y$ is the minor-axis direction. The quantity $\Delta y$ was taken to be the approximate extent of the region around the major axis 
over which data were obtained. For NGC 185, we also took into account that the observed stars are on average somewhat offset by $\delta y \approx-1.5$ from the major axis (Figure 1). No luminosity weighting was applied in calculation of the binned model predictions, since the observed stars are distributed approximately homogeneously in each bin.

The value of $M / L$ was chosen to best fit the folded $V_{\mathrm{rms}}$ profile near the galaxy center, which in practice was chosen to be the region inside $4^{\prime}$. We determine mass-to-light ratios of $M / L_{V}=4.2 \pm 0.6$ and $M / L_{V}=4.6 \pm 0.6$ for NGC 147 and NGC 185, respectively. The value of $k$ in our models was chosen to best fit the rotation curve at all available radii. The parameters of the best-fitting models are listed in Table 3 . Figure 5 compares the corresponding model predictions to the observed kinematical profiles. We discuss the $M / L$ values further in Section 4.4.

For NGC 147, the predicted profile provides a statistically acceptable match to the $V_{\text {rms }}$ data $\left(\chi^{2}=3.8\right.$ for 8 degrees of freedom or $\chi_{v}^{2}=0.5$ ). Although the $M / L$ was chosen to only optimize the fit near the center, the predicted profile fits the full observed profile out to the last measured data point. Hence, even data out to $\sim 7.3 r_{\text {eff }}$ provide no evidence for an increase in $M / L$ with radius. By contrast, for NGC 185 , the predicted $V_{\text {rms }}$ profile does not provide a statistically acceptable match to the data. The fit is reasonable inside $4^{\prime}$, since the $M / L$ was chosen to optimize the fit there. However, for the data points outside of this radius the observed weighted average $V_{\mathrm{rms}}=23.0 \pm 1.1 \mathrm{~km} \mathrm{~s}^{-1}$. The average model prediction at these radii is $V_{\mathrm{rms}}=20.6 \mathrm{~km} \mathrm{~s}^{-1}$. Therefore, the observations exceed the model predictions at large radii with $2.2 \sigma$ significance. The most natural interpretation of this result is that the $M / L$ of NGC 185 is increasing with radius, although tides may also produce this effect (see Section 4.5).

The predicted rotation curves for both galaxies provide a statistically acceptable match to the data $\left(\chi^{2}=11.9\right.$ and 7.6, for 8 and 7 degrees of freedom, for NGC 147 and NGC 185, respectively). However, inspection of the predicted rotation curves shows that they may be slightly too high at small radii and slightly too low at large radii. This could easily be improved by choosing $k$ to be an increasing function of radius. This would be no less arbitrary than choosing it to have a constant value as a function of radius, as we have done here. If the model also provides a statistically acceptable fit to $V_{\text {rms }}$, as is the case for NGC 147, then this guarantees that the velocity dispersion $\sigma$ is fit as well. By contrast, for NGC 185 , an increase of $M / L$ with radius might be a more natural way to improve the fit to the rotation curve, given the $V_{\text {rms }}$ results. The best-fitting $k$ values in Table 3 indicate that while both galaxies have significant rotation rates, neither galaxy rotates fast enough to account for its flattening. Hence, the velocity dispersion tensors must be anisotropic, in agreement with our conclusion from Section 3.1.

The fact that NGC 147 is well fit by a constant- $M / L$ twointegral model does not mean that it is not embedded in a dark halo. The profile of $V_{\text {rms }}$ with radius is almost flat. So it is likely that models with a logarithmic gravitational potential can fit the data equally well. Conversely, the fact that NGC 185 is not well fit by constant $M / L$ two-integral model does not mean that it necessarily must have a dark halo. It may be possible to fit the data with a constant $-M / L$ model that has a different velocity dispersion anisotropy than assumed here. Alternatively, our assumption of dynamical equilibrium may be incorrect for NGC 185 at large radius, and we discuss the possibility of tidal interactions in Section 4.5. In principle, it is possible to constrain the velocity dispersion anisotropy independent of the assumed gravitational potential by using information on deviations of the line-of-sight velocity distributions from Gaussians (e.g., Carollo et al. 1995; Rix et al. 1997). To further explore these issues, we intend to use the discrete Schwarzschild method of Chaname et al. (2008) to construct dynamical models with three-integral distribution function, including a dark halo (i.e., $M / L$ varying with radius), which will avoid spatial binning of the data. Such models will not necessarily yield smaller error bars or better constraints on quantities of interest (such as the mass-to-light ratio or the presence of a dark halo), given the limited number of stars available to trace the kinematics. However, they will have the benefit of yielding more rigorous error bars and constraints, which include, e.g., modeling uncertainties due to the unknown velocity anisotropy and radial mass-to-light ratio profiles of these systems.

\subsection{Mass-to-Light Ratios}

We compare our inferred $M / L$ ratios with stellar population models to determine whether or not non-baryonic dark matter is needed to explain our observed kinematics. Despite our restrictive model assumptions, our resulting $M / L$ values are likely very robust. Both van der Marel \& van Dokkum (2007b) and Cappellari et al. (2006) have compared the results of several dynamical modeling studies, showing that different modeling approaches produced consistent $M / L$ ratios to within a systematic accuracy of $\sim 5 \%$. This is true despite the use of varying data sets (long-slit and integral field data) and modeling approaches (two- and three-integral models, and models with and without dark halos). In the context our models, we have further explored the robustness of our inferred $M / L$ values by constructing models with a range of inclinations (from edge-on to inclinations corresponding to models as flat as $\left.q_{t}=0.4\right)$. Consistent with previous studies, we find that both the $M / L$ values and the predicted profiles of $V_{\text {rms }}$ with radius are insensitive to the assumed inclination (well within the error bars). We also explored models with different ways of extrapolating Kent's (1987) surface brightness data to larger radii. Again, we found the effect on the $M / L$ values and the predicted profiles of $V_{\text {rms }}$ with radius to be well within the error bars.

We first compare our $M / L$ values to those of de Rijcke et al. (2006) who modeled NGC 147 and NGC 185 using a threeintegral method. For the comparison, we transform our $M / L_{V}$ values to the $B$ band. The $B$-band values were obtained from the modeled $V$-band values using the galaxy $(B-V)_{0}$ color (Table 3 ) and the solar color $(B-V)_{\odot}=0.65$. The de Rijcke et al. value for NGC $147, M / L_{B}=4.0_{-2.4}^{+3.2}$, is consistent with our result. However, their value for NGC $185, M / L_{B}=3.0_{-0.7}^{+1.0}$, is lower than our result at $1.5 \sigma$ significance. The underlying reason for this may be that NGC 185 has an increasing $M / L$ with radius, as suggested by Figure 5 . The best-fitting $M / L$ is then likely to depend on the radial extent of the data, which is larger for our data set than for the integrated-light measurements of de Rijcke et al. (2006).

Figure 6 shows the mass-to-light ratio $M / L_{B}$ for each $\mathrm{dE}$ versus the extinction-corrected color $(B-V)_{0}$. We compare these measurements to the stellar population predictions of S. Charlot \& A. G. Bruzual (2007, private communication) which is an updated version of their Bruzual \& Charlot (2003) models. The $[\mathrm{Fe} / \mathrm{H}]$ metallicities, determined in Section 3.2, are $Z=0.0015([\mathrm{Fe} / \mathrm{H}]=-1.1)$ for NGC 147 and $Z=0.0009$ $([\mathrm{Fe} / \mathrm{H}]=-1.3)$ for NGC 185 which assumes a solar 

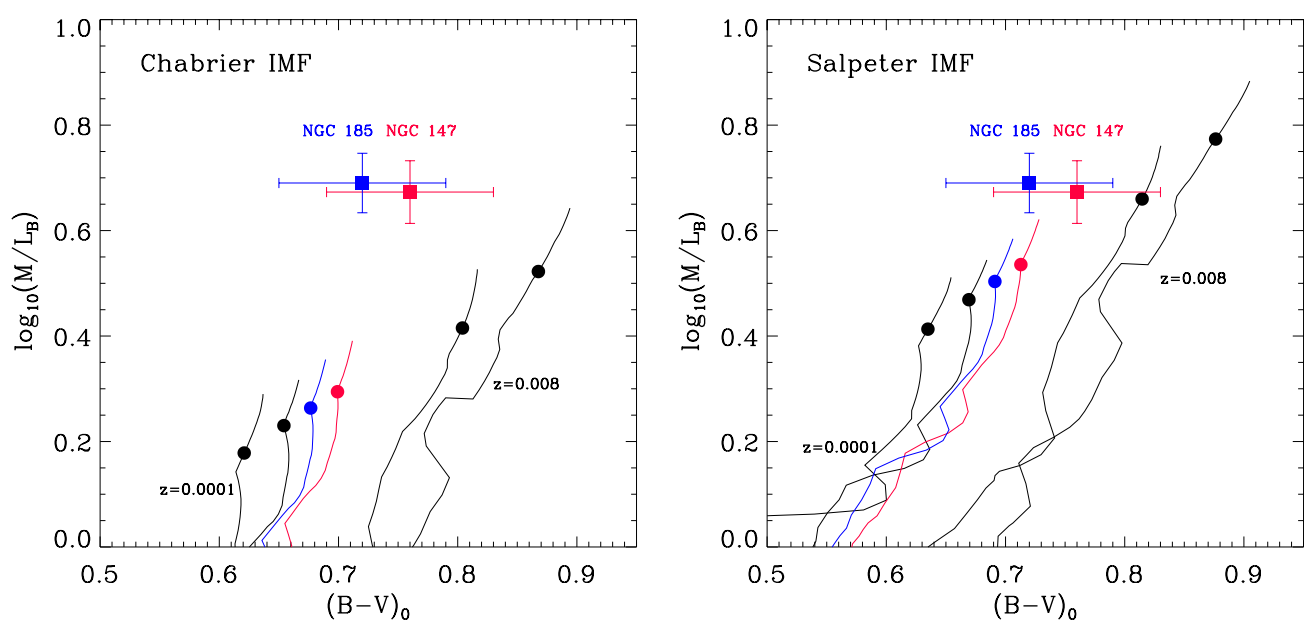

Figure 6. $M / L_{B}$ vs. extinction corrected color $(B-V)_{0}$. Observed values for NGC 147 (red) and NGC 185 (blue) are shown as data points with error bars. Curves show model predictions from S. Charlot \& A. G. Bruzual (2007, private communication) for the IMFs from Chabrier (2003; left) and Salpeter (1955; right). Each curve is for a fixed metallicity $Z$ and connects the predictions of simple stellar populations of fixed age. Solid circles indicate ages of $10 \mathrm{Gyr}$ for each metallicity. The predicted curves extend up to the age of the Universe (13.73 Gyr; Spergel et al. 2007). Black curves are, from left to right, $Z=0.0001,0.0004,0.004$, and 0.008. The red and blue curves shows the interpolation of these curves to the metallicity appropriate for NGC 147 and NGC 185, respectively. We note for the preferred Chabrier IMF, the inferred $M / L_{B}$ ratios of these $\mathrm{dE}$ galaxies cannot be explained via their stellar content alone.

(A color version of this figure is available in the online journal.)

composition of $Z_{\odot}=0.0189$ (Anders \& Grevesse 1989). The predictions at these metallicities are overplotted in Figure 6 for both the initial mass functions (IMFs) of Chabrier (2003) and Salpeter (1955) over the range from 0.1 to $100 M_{\odot}$. For the Chabrier IMF, the maximum $M / L_{B}$ ratio at the metallicity of NGC 147 and NGC 185 is attained for a population as old as the universe (13.73 Gyr; Spergel et al. 2007) with values $M / L_{B, \max }=2.5$ and 2.3 , respectively. This is not as high as observed, so for the Chabrier IMF one must invoke the presence of non-baryonic dark matter to fit the observed $M / L_{B}$ values. The Salpeter IMF tracks, while closer to the observations, are only marginally consistent with observations for very old stellar populations. Stellar population studies suggest significant intermediate-age stellar populations in both dEs (Butler \& Martínez-Delgado 2005), thus the Salpeter IMF also implies the presence of dark matter. The Chabrier IMF is now generally preferred over the Salpeter IMF, as the latter has too many stars at low masses to properly fit real populations (e.g., Bell \& de Jong 2000; Chabrier 2003). Therefore, the high observed $M / L$ values in NGC 147 and NGC 185 must be due at least in part to the presence of dark matter.

Figure 7 shows $M / L_{B}$ versus the velocity dispersion $\sigma$. For NGC 147 and NGC 185, we use along the abscissa the weighted average $V_{\text {rms }}$ inside $4^{\prime}$, as listed in Table 1 . For comparison, we show in Figure 7 the sample of (giant) elliptical galaxies compiled by van der Marel $\&$ van Dokkum (2007b). The latter follow a tight linear relation of the form $M / L_{B} \propto \sigma$. The $\mathrm{dE}$ galaxies NGC 147 and NGC 185 clearly do not follow this relation. This result is not specific to these two $\mathrm{dE}$ galaxies. For comparison, we plot also the results for NGC 205 from Geha et al. (2006), and for six dE galaxies in Virgo from Geha et al. (2002). These all fall in the same region of the plot. The same distinction between dwarf and giant ellipticals is also revealed by plots of the fundamental plane; see e.g., the plot of $\kappa_{3}$ versus $\kappa_{1}$ in Geha et al. (2003). A traditional interpretation of this is that it indicates a fundamental distinction between dwarf and giant ellipticals. However, Graham \& Guzman (2003) have proposed more recently that there is actually a continuous sequence between the two, with the upturn in $M / L$ toward

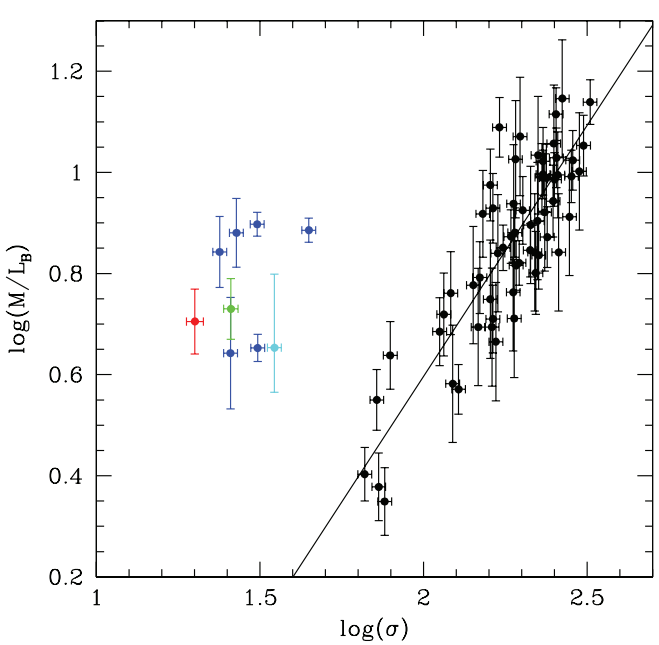

Figure 7. $M / L_{B}$ vs. velocity dispersion $\sigma$. Observed values for NGC 147 and NGC 185 are shown in red and green, respectively. Cyan and blue data points are other dE galaxies from de Rijcke et al. (2006) and Geha et al. (2002), respectively. Black data points are giant ellipticals from the compilation of van der Marel \& van Dokkum (2007b).

(A color version of this figure is available in the online journal.)

$\mathrm{dE}$ galaxies driven by the fact that their Sérsic $n$ parameter is lower than for giant ellipticals. Either way, it is clear from Figure 7 that $\mathrm{dE}$ galaxies have $M / L$ values that are similar to those of intermediate- to high-luminosity giant ellipticals. The latter are redder and have higher metallicity (and quite possibly older stellar populations) than NGC 147 and NGC 185. A natural explanation for the observed $M / L$ of NGC 147 and NGC 185 is that these galaxies have a significant dark matter component, consistent with our conclusions from the comparison to population synthesis models.

\subsection{A Bound Pair?}

Given our velocity and mass estimates, we revisit the question of whether or not NGC 147 and NGC 185 form a gravitationally 
bound pair. The angular distance between NGC 147 and NGC 185 is $58^{\prime}=10 \mathrm{kpc}$. Assuming distances measured by McConnachie et al. (2005), NGC 147 and NGC 185 are separated by a line-of-sight distance of $59 \mathrm{kpc}$, and thus a total physical separation of $r=60 \mathrm{kpc}$. For two point masses to be gravitationally bound, their potential energy must exceed the kinetic energy of the system (Davis et al. 1995; van den Bergh 1998). This leads to the criterium $b \equiv\left(2 G M_{\text {sys }} / r \Delta v^{2}\right)>1$ for a system to be bound, where $M_{\text {sys }}$ is the combined system mass. We measure, in Section 3, a radial velocity difference between these two dEs of $\Delta v=10.7 \pm 1.3 \mathrm{~km} \mathrm{~s}^{-1}$. Approximating our $\mathrm{dE}$ galaxies as point masses, we calculate the criteria for these two objects to be bound as $b=1.6 \pm 1.1$. This criterion suggests that, in the absence of transverse motion, NGC 147 and NGC 185 are a gravitationally bound pair, in agreement with van den Bergh (1998). However, the large error on this quantity, in addition to the likely non-zero transverse motions of these two satellites, leaves wide open the possibility that these are not in fact bound.

NGC 147 and NGC 185 are satellites of M 31. Our equilibrium models assume that the outer kinematics of these dEs are not affected by tidal interactions with $\mathrm{M} 31$, or each other. This is not the case for the third dE in the Local Group, NGC 205, which is in the process of being tidal stripped at radii beyond $4^{\prime} .5=1 \mathrm{kpc}$ by M 31. To confirm our equilibrium assumption for NGC 147 and NGC 185, we calculate their instantaneous tidal radius in the rotating frame by again approximating these galaxies as point sources. We assume a total mass for M 31 between 0.8 and $1.6 \times 10^{12} M_{\odot}$ (Klypin et al. 2002; Seigar et al. 2008). We determine a tidal (Jacobi) radius between 10 and $12 \mathrm{kpc}$ (greater than $25 r_{\text {eff }}$ ) in each $\mathrm{dE}$. For comparison, the tidal radius of the interacting dE NGC 205 is $0.7 \mathrm{kpc}$. The internal kinematic profiles of NGC 147 and NGC 185 are unlikely to be currently affected by tidal interactions with M 31, at the distances probed by the data presented here. However, these calculations do not preclude the possibility of past tidal interactions, either between these two galaxies or with M 31, and it is yet possible that there are unbound stars in our kinematic sample. The regularity and bi-symmetry of our kinematic data for NGC 147 suggest that any contamination is minimal. In NGC 185 , the upturn of $V_{\mathrm{rms}}$ profile over our models at large radius may either be interpreted as an increasing $M / L$ ratio with radius, or possible tidal contamination. Deeper imaging and kinematic observations are needed, particularly for NGC 185 , to determine to what extent these dEs are affected by tidal interactions at radii beyond $8 r_{\text {eff }}$ in these galaxies.

\section{CONCLUSIONS}

We have presented mean velocity, velocity dispersion, and metallicity profiles for the Local Group dE galaxies NGC 147 and NGC 185 based on Keck/DEIMOS spectroscopic observations of 520 and 442 member RGB stars in each dE, respectively (Tables 6 and 7). The profiles represent the most extensive spectroscopic radial coverage for any $\mathrm{dE}$ galaxy, extending to a projected distance of eight half-light radii $\left(14^{\prime} \sim 8 r_{\text {eff }}\right)$. In contrast to previous results, we find that both dEs have significant rotation velocities which contributes to the observed flattened shapes of each galaxy. Our two-integral dynamical modeling suggests that the observed rotation velocities cannot fully explained the observed shapes and that some anisotropic velocity dispersion is required. Our modeling estimates $M / L$ ratios of $M / L_{V}=4.2 \pm 0.6$ and $M / L_{V}=4.6 \pm 0.6$ for NGC 147 and NGC 185 , respectively, which is in excess of that expected from stellar populations alone. Thus, some dark matter is required to fully explain the observe kinematics.

The mean velocity profiles of NGC 147 and NGC 185 suggest that rotation may be far more prevalent in $\mathrm{dE}$ galaxies than previously assumed. We conclude that these Local Group dEs are rotationally supported; however, if placed at the distance of the Virgo or Fornax Clusters, integrated-light observations such as those of Geha et al. (2003) would have concluded that these dEs have little to no rotation. It is not guaranteed that the Local Group dE galaxies had similar formation mechanisms as those residing in the much denser environments of, e.g., the Virgo or Fornax clusters. However, this is certainly plausible, given that these galaxies share similar fundamental plane properties, as defined by the photometric and kinematic properties inside $r_{\text {eff }}$ (see, e.g., Figure 7). Our results then indicate that cluster dEs may also have significant rotation velocities that are manifest only at larger radii than current observations allow. If true, this significantly modifies the observational constraints under which $\mathrm{dEs}$ can form. The observations presented here open the door for formation mechanisms in which dEs are transformed or stripped versions of gas-rich rotating progenitor galaxies.

We thank S. Demers and P. Battinelli for kindly providing their photometric catalogs before publication. We also kindly thank Michael Rich for sharing Keck observing time with part of this project. Support for this work was provided in part by NASA through Hubble Fellowship grant HST-HF01233.01 awarded to E.N.K. by the Space Telescope Science Institute, which is operated by the Association of Universities for Research in Astronomy, Inc., for NASA under contract NAS 5-26555.

\section{REFERENCES}

Aguerri, J. A. L., \& González-García, A. C. 2009, A\&A, 494, 891

Anders, E., \& Grevesse, N. 1989, Geochim. Cosmochim. Acta, 53, 197

Baade, W. 1944, ApJ, 100, 147

Battinelli, P., \& Demers, S. 2004a, A\&A, 418, 33

Battinelli, P., \& Demers, S. 2004b, A\&A, 417, 479

Beasley, M. A., Cenarro, A. J., Strader, J., \& Brodie, J. P. 2009, AJ, 137, 5146 Beasley, M. A., Strader, J., Brodie, J. P., Cenarro, A. J., \& Geha, M. 2006, AJ, 131,814

Bell, E. F., \& de Jong, R. S. 2000, MNRAS, 312, 497

Bender, R., Burstein, D., \& Faber, S. M. 1992, ApJ, 399, 462

Bender, R., Paquet, A., \& Nieto, J.-L. 1991, A\&A, 246, 349

Bruzual, A. G., \& Charlot, S. 2003, MNRAS, 344, 1000

Butler, D. J., \& Martínez-Delgado, D. 2005, AJ, 129, 2217

Cappellari, M., et al. 2006, MNRAS, 366, 1126

Carollo, C. M., de Zeeuw, P. T., van der Marel, R. P., Danziger, I. J., \& Qian, E. E. 1995, ApJ, 441, L25

Chabrier, G. 2003, PASP, 115, 763

Chanamé, J., Kleyna, J., \& van der Marel, R. 2008, ApJ, 682, 841

Chilingarian, I. V., Prugniel, P., Sil'Chenko, O. K., \& Afanasiev, V. L. 2007, MNRAS, 376, 1033

Choi, P. I., Guhathakurta, P., \& Johnston, K. V. 2002, AJ, 124, 310

Coccato, L., et al. 2009, MNRAS, 394, 1249

Davidge, T. J. 2005, AJ, 130, 2087

Davies, R. L., Efstathiou, G., Fall, S. M., Illingworth, G., \& Schechter, P. L. 1983, ApJ, 266, 41

Davis, D. S., Bird, C. M., Mushotzky, R. F., \& Odewahn, S. C. 1995, ApJ, 440, 48

de Rijcke, S., Michielsen, D., Dejonghe, H., Zeilinger, W. W., \& Hau, G. K. T. 2005, A\&A, 438, 491

de Rijcke, S., Prugniel, P., Simien, F., \& Dejonghe, H. 2006, MNRAS, 369, 1321

de Vaucouleurs, G., et al. 1991, Third Reference Catalogue of Bright Galaxies (Berlin: Springer)

Dekel, A., \& Silk, J. 1986, ApJ, 303, 39

Faber, S. M., et al. 2003, Proc. SPIE, 4841, 1657

Ferguson, H. C., \& Binggeli, B. 1994, A\&AR, 6, 67 
Ferguson, H. C., \& Sandage, A. 1991, AJ, 101, 765

Geha, M., Guhathakurta, P., Rich, R. M., \& Cooper, M. C. 2006, AJ, 131, 332

Geha, M., Guhathakurta, P., \& van der Marel, R. P. 2002, AJ, 124, 3073

Geha, M., Guhathakurta, P., \& van der Marel, R. P. 2003, AJ, 126, 1794

Geha, M., Guhathakurta, P., \& van der Marel, R. P. 2005, AJ, 129, 2617

Gilbert, K. M., et al. 2006, ApJ, 652, 1188

Gonçalves, D. R., Magrini, L., Leisy, P., \& Corradi, R. L. M. 2007, MNRAS, 375,715

Graham, A. W., \& Guzmán, R. 2003, AJ, 125, 2936

Guhathakurta, P., et al. 2005, arXiv:astro-ph/0502366

Han, M., Hoessel, J. G., Gallagher, J. S. III, Holtsman, J., \& Stetson, P. B. 1997, AJ, 113, 1001

Howley, K. M., Geha, M., Guhathakurta, P., Montgomery, R. M., Laughlin, G., \& Johnston, K. V. 2008, ApJ, 683, 722

Jerjen, H., Kalnajs, A., \& Binggeli, B. 2000, A\&A, 358, 845

Jorgensen, I. 1994, PASP, 106, 967

Kalirai, J. S., et al. 2006, ApJ, 648, 389

Kent, S. M. 1987, AJ, 94, 306

Kirby, E. N., Guhathakurta, P., \& Sneden, C. 2008, ApJ, 682, 1217

Klypin, A., Zhao, H., \& Somerville, R. S. 2002, ApJ, 573, 597

Kormendy, J., Fisher, D. B., Cornell, M. E., \& Bender, R. 2009, ApJS, 182, 216

Lisker, T., Grebel, E. K., \& Binggeli, B. 2006, AJ, 132, 497

Martínez-Delgado, D., Aparicio, A., \& Gallart, C. 1999, AJ, 118, 2229

Mastropietro, C., Moore, B., Mayer, L., Debattista, V. P., Piffaretti, R., \& Stadel, J. 2005, MNRAS, 364, 607

Mayer, L., Governato, F., Colpi, M., Moore, B., Quinn, T., Wadsley, J., Stadel, J., \& Lake, G. 2001, ApJ, 559, 754

McConnachie, A. W., Irwin, M. J., Ferguson, A. M. N., Ibata, R. A., Lewis, G. F., \& Tanvir, N. 2005, MNRAS, 356, 979
Moore, B., Lake, G., \& Katz, N. 1998, ApJ, 495, 139

Pedraz, S., Gorgas, J., Cardiel, N., Sánchez-Blázquez, P., \& Guzmán, R. 2002, MNRAS, 332, L59

Rix, H.-W., de Zeeuw, P. T., Cretton, N., van der Marel, R. P., \& Carollo, C. M. 1997, ApJ, 488, 702

Rutledge, G. A., Hesser, J. E., \& Stetson, P. B. 1997a, PASP, 109, 907

Rutledge, G. A., et al. 1997b, PASP, 109, 883

Sage, L. J., Welch, G. A., \& Mitchell, G. F. 1998, ApJ, 507, 726

Salpeter, E. E. 1955, ApJ, 121, 161

Schlegel, D. J., Finkbeiner, D. P., \& Davis, M. 1998, ApJ, 500, 525

Seigar, M. S., Barth, A. J., \& Bullock, J. S. 2008, MNRAS, 389, 1911

Simien, F., \& Prugniel, P. 2002, A\&A, 384, 371

Simon, J. D., \& Geha, M. 2007, ApJ, 670, 313

Sohn, S. T., et al. 2007, ApJ, 663, 960

Spergel, D. N., et al. 2007, ApJS, 170, 377

Strigari, L. E., Bullock, J. S., Kaplinghat, M., Simon, J. D., Geha, M., Willman, B., \& Walker, M. 2008, Nature, 454, 1096

Tremblay, B., \& Merritt, D. 1995, AJ, 110, 1039

van den Bergh, S. 1998, AJ, 116, 1688

van der Marel, R. P., \& Anderson, J. 2009, ApJ, submitted (arXiv: astro-ph/0905.0638)

van der Marel, R. P., Evans, N. W., Rix, H.-W., White, S. D. M., \& de Zeeuw, T. 1994, MNRAS, 271, 99

van der Marel, R. P., \& van Dokkum, P. G. 2007a, ApJ, 668, 738

van der Marel, R. P., \& van Dokkum, P. G. 2007b, ApJ, 668, 756

van Zee, L., Barton, E. J., \& Skillman, E. D. 2004, AJ, 128, 2797

VandenBerg, D. A., Bergbusch, P. A., \& Dowler, P. D. 2006, ApJS, 162, 375

Young, L. M., \& Lo, K. Y. 1997, ApJ, 476, 127 